This manuscript in post-review format was accepted for publication in Acta Geotechnica and can be cited as follows

Karim Ehab Moustafa Kamel, Jean-Baptiste Colliat, Pierre Gerard, Thierry J. Massart, Comparison of advanced discretization techniques for image-based modelling of heterogeneous porous rocks, Acta Geotechnica, In Press, 2019, https://doi.org/10.1007/s11440-019-00798-7 


\title{
Comparison of advanced discretization techniques for image-based modelling of heterogeneous porous rocks
}

\author{
Karim Ehab Moustafa Kamel . \\ Jean-Baptiste Colliat • Pierre Gerard . \\ Thierry J. Massart
}

Received: date / Accepted: date

\begin{abstract}
This contribution presents an assessment of computational techniques enabling automated simulations of complex porous rocks microstructures based on 3D imaging techniques. A subset of a CT-scanned sandstone sample is used to compare the results obtained by two advanced discretization frameworks. Raw scan results are processed by a level set-based segmentation technique to produce smooth geometries prone to finite element discretizations. A recently developed technique is outlined for conforming mesh generation for complex porous geometries described implicitly by functions. This allows generating high quality tetrahedral meshes with selective refinement. Next to this, a technique that uses a kinematic enrichment by incompatible modes to represent the heterogeneous geometry is explained. Both techniques use the same implicit geometry as main input for the simulations. Mechanical simulations are conducted on a subset of a scanned sample of a sandstone under triaxial loading conditions for isotropic compressive loading and for loading conditions involving a stress deviator. The results are compared and discussed based on local stress distributions and on a Mohr-Coulomb criterion with tensile cut-off. The results show that both discretization strategies yield complementary tools and allow envisioning automated simulations based on raw CT scan data for porous rocks exhibiting complex pore space morphologies.
\end{abstract}

Keywords Rock Mechanics · Porous Rocks · Automated Meshing · Finite Elements · Embedded Discontinuities · CT scan · Image-based Modelling

K. Ehab Moustafa Kamel

E-mail: kehabmou@ulb.ac.be

J. B. Colliat

E-mail: jean-baptiste.colliat@univ-lille.fr

P. Gerard

E-mail: piergera@ulb.ac.be

T. J. Massart

E-mail: thmassar@ulb.ac.be

Tel.: +32 26502742 , Fax : +3226502789

BATir, Building, Architecture \& Town Planning, Université Libre de Bruxelles (ULB), CP 194/2, 1050 - Brussels, Belgium

Laboratoire de mécanique, multiéchelle, multiphysique, Université de Lille, Centrale Lille, CNRS, France. 


\section{Context}

The mechanical and fluid transport behaviour of porous geomaterials is strongly dependent on their microstructure, and in particular on the morphological properties of their pore or fracture networks. Furthermore, these microstructural features may evolve as a result of mechanical loading, which induces further couplings between the mechanical response and the transport properties.

As a result, many research efforts have been devoted in the past to investigate the link between the microstructure of geomaterials (in the broad sense) and their overall properties. Initially, many of these contributions dealt with the effect of degradation and cracking evolution on the mechanical response of geomaterials. This was achieved using various techniques such as micromechanically-inspired models [1], discrete element modelling [2] or RVE computations [3]. Fluid transport properties and their link to cracking evolution were also scrutinized using micromechanically-inspired models [1] and multiscale techniques [4, 5].

With the development of experimental imaging techniques at different scales, the investigations on the effect of microstructural features on the overall behaviour received even more attention.

Focusing on scanning techniques allowing to obtain the microstructural phase arrangement, computed tomography (CT) nowadays allows addressing a variety of scales (micro-tomography or at higher scales) and of geomaterials such as soils [6], rocks [7] or mixtures [8]. Such investigations are devoted to their mechanical behaviour [7, 8], to their transport properties [9]; or to coupled processes [10].

Early efforts in the use of 3D images for analysis and modelling of rocks behaviour started with an effort reconstructing a 3D granite mesostructure (at the scale of a full lab specimen), based on an iterative milling and scanning system presented in [7]. The procedure was used to conduct 3D Brazilian test simulations on a real mesostructural geometry by means of a finite difference scheme. Subsequent efforts at similar (large) scales were devoted to jointed rocks (sandstone) [11], in which computed tomography was used to analyse and model the effect of joints shape on the mechanical behaviour; and to feed soil-rock mixture 2D simulations [8].

Experimental efforts in the field of rock mechanics to capture microstructural features used micro-tomography $(\mu \mathrm{CT})$ to investigate the microstructural changes in the pore space during unconfined compression tests in Gosford sandstone [12]. This was achieved by scanning samples at different loading levels prior to failure in order to link the pore structure evolution to the macroscopic energy dissipation. In situ $\mu \mathrm{CT}$ observations of porosity evolutions under triaxial tests were also investigated for calcarenite in [13]. The relationship between specific surface and porosity in ten different types of sandstone was scrutinized in [14] by $\mu \mathrm{CT}$ in order to build modified Carman-Kozeny equations for sandstones; and real time (in situ) $\mu \mathrm{CT}$ triaxial experiments were performed in sandstone to assess the effect of chemical corrosion in [15].

With the availability of the detailed 3D microstructural geometry, $\mu \mathrm{CT}$ also allowed feeding microstructural mechanical computations making direct use of the image information. Such observations were mostly used to feed 3D DEM simulations on sandstones at the RVE and at the sample scales [3]. Strength anisotropy was scrutinized in Berea sandstone together with an $\mu \mathrm{CT}$ assessment of the porosity levels to feed 2D DEM simulations [16]. Additional efforts addressed the up- 
scaling of transport properties by using multiscale approaches using finite element macroscopic computations fed by a lattice Boltzmann framework based on 3D $\mu \mathrm{CT}$ images $[9,17]$.

Next to the direct use of $3 \mathrm{D}$ images, $\mu \mathrm{CT}$ observations were also exploited to reconstruct equivalent geometrical models which can subsequently be used in finite element simulations. Such a reconstruction strategy was proposed in [18] for porous rocks (sandstone). It was next extended in [19] using simulated annealing, leading to models that do not consist of the scanned geometry itself, but rather of equivalent geometries reproducing the essential features identified in the scans. These techniques were also applied in [20] to triaxial tests with comparisons to experiments.

These previous contributions in the literature show that there is an interest in addressing the simulation of microstructural processes in porous rocks using directly the $3 \mathrm{D}$ images obtained by $\mu \mathrm{CT}$. However, the discretization of such geometries may quickly become extremely complex. The purpose of the present contribution is to compare two novel discretization strategies that allow building models in an automated way based on a raw $3 \mathrm{D} \mu \mathrm{CT}$ image. The first of these discretization strategies consists of a recent extension of the meshing tool relying on the Persson-Strang analogy [21, 22] to build a high quality conforming tetrahedral mesh. It relies on an implicit description of the geometry of the solid phase and of the pore space of a porous rock. The second strategy is based on the projection of such an implicit geometry on an unstructured tetrahedral mesh, with an incompatible kinematic enrichment defined at the element level to account for the heterogeneity of phases. The comparison will here be performed through mechanical simulation on a subset of the CT-scanned image of a sandstone [23].

To build this comparison, this contribution will be structured as follows. Section 2 will outline the pre-processing required from the raw $\mu \mathrm{CT}$ scan data (segmentation and smoothing) to provide the implicit geometrical information for the discretization tools. Section 3 will next detail the procedures followed by each strategy to construct the discretization based on the implicit geometry devised in Section 2 from the raw $\mu \mathrm{CT}$ scan data. Section 4 will compare the results obtained by both strategies for a sandstone $\mu \mathrm{CT}$ scan sample subjected both to a pure isotropic triaxial stress state and to a triaxial stress state involving a stress deviator. Finally the results will be discussed in Section 5, before conclusions are drawn in Section 6.

\section{Problem statement and segmentation}

In the context of image-based modelling of heterogeneous porous rocks, the porous microstructure can be obtained either by reconstruction of equivalent geometrical models from the exploitation of $3 \mathrm{D}$ images or by virtual generation according to morphological quantitative features [24, 25]. When dealing with image-based models, the interfaces separating the pore space from the solid phase can be described either explicitly by means of triangulated surfaces in 3D, or implicitly by means of level-set functions and/or distance fields. The latter approach offers the ability to model and handle geometries with arbitrarily complex features. When using level sets, a curve $\Phi$ in 2D, respectively a surface in $3 \mathrm{D}$, can be represented implicitly 

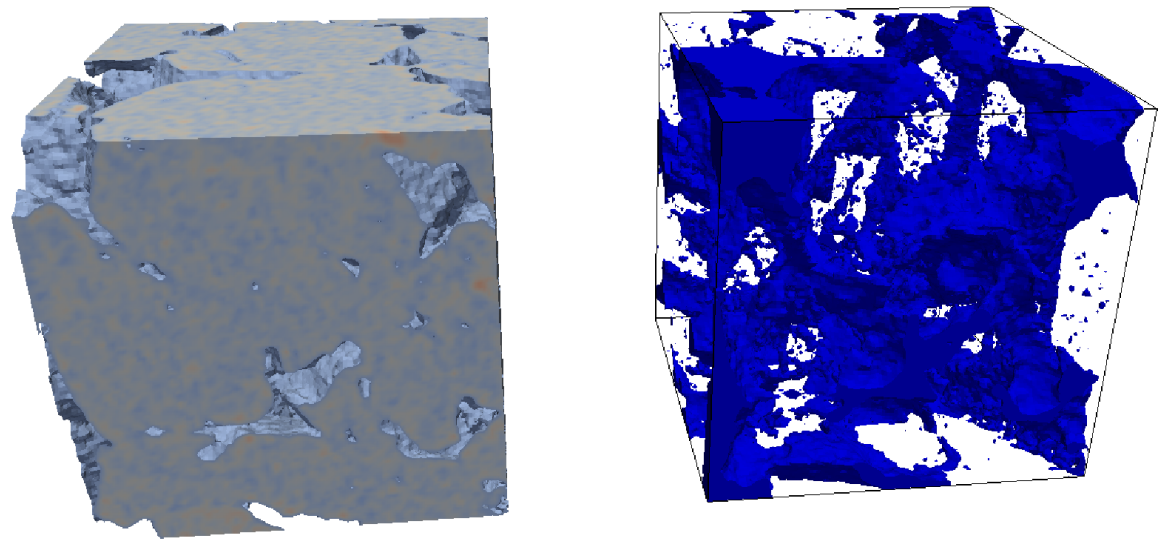

Fig. 1: Subset of Vosges sandstone sample obtained by X-ray tomography from Cong Hu's thesis [23] : solid part (left) and pore space (right) of the sandstone

by means of the level sets of functions of spatial coordinates $\mathbf{x}$ denoted $L S(\mathbf{x}, \ldots)$ :

$$
\Phi \equiv L S(\mathbf{x}, \ldots)=k
$$

where $k$ represents the iso-value of the function.

A well-known example of level set function consists of the gray-scale density map obtained by X-ray CT scans increasingly used in the context of rock mechanics. To exploit properly imaging techniques, the segmentation process is however a crucial step. Indeed, it must accurately reflect the complexity of the geometry at hand, be able to identify the different phases of the material, and make the results usable in the subsequent processes. The sample used in this paper to illustrate the comparison between the two selected discretization methods is a subset of the CT scan of a dry Vosges sandstone obtained by X-ray tomography as part of Cong Hu's PhD thesis [23]. The scan resolution is 5 microns per voxel for a geometry size of 100x100x100 voxels (see Figure 1).

Since the pore space ( $18.04 \%$ of total porosity) of the studied sandstone sample is very strongly connected (about $99 \%$ ), the occluded porosities represent only very small cavities. These may require a high level of refinement in the discretization step if they are still taken into account in the segmentation process. Therefore, assuming that their impact on the simulation results discussed in this paper will be negligible, they will be removed from the voxel grid before segmentation in order to reduce computation times. To achieve this, a geodesic reconstruction [26] is applied to the given grayscale image using as a mask the 6 boundaries of the scan sample, so that only the connected porosities remain.

For the image segmentation, an automated thresholding by Otsu's method [27] is suitable when the histogram of the grayscale of the images shows two distinct normal distributions. Otherwise, overlapping will induce thresholding management that may atrophy part of the information. In addition, this method often requires the application of a Gaussian filter to smoothen the interfaces, a required step to obtain a mesh-prone resulting geometry. Among the other available segmentation techniques, the level-sets-based methods correspond to a class of deformable mod- 

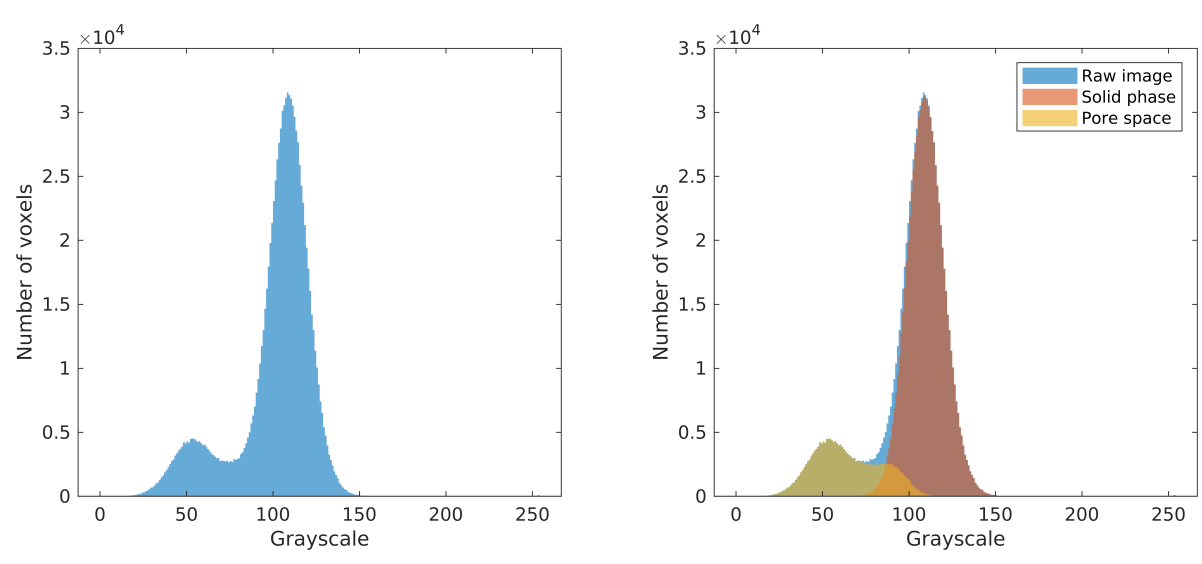

Fig. 2: (a) Histogram of the geometry, (b) Decomposition of the histogram in two phases taking into account the overlapping with the level-set segmentation method
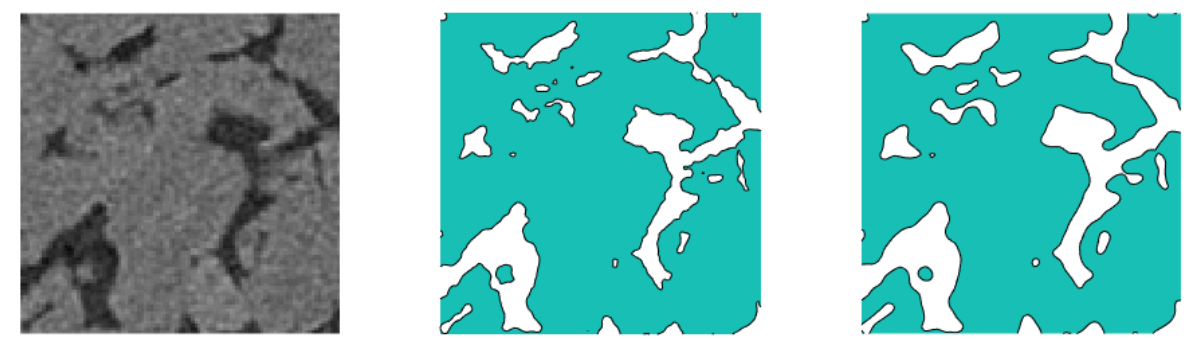

Fig. 3: Comparison between : (a) a slice of the micro-CT scan, (b) thresholding process obtained by Otsu's method at 76 grayscale value, (c) level-set segmentation

els in which the desired shape is obtained by propagating an interface represented by $2 \mathrm{D}$ iso-contours (3D iso-surfaces) $[28,29,30]$ giving thereby smoothed contours.

Even if in this case two peaks can be distinguished relatively clearly, a level-set segmentation method based on Bernard's work [31] will be used. In this chosen method, the implicit function is modeled as a continuous parametric function expressed on a B-spline basis, that allows obtaining a smooth level-set function and a satisfactory segmentation result of the image given in input; even if it is noisy. Figure 3 illustrates a comparison between an image built from the raw micro-CT scan data, using the thresholding by the Otsu method, and using the level-set segmentation for a $2 \mathrm{D}$ slice.

The segmentation process was applied here for each of the slices of a raw 3D CT scan, before being stacked to form the whole result in 3D as illustrated in Figure 4.

The discretization methods presented in the following sections require the use of signed distance functions which is a particular choice for level-set functions [32, 33]. The signed distance function of $\Phi$ is a function $D S(\Phi(\mathbf{x}))$ with the value of the signed euclidian distance from $\mathbf{x}$ to $\Phi$. Here, the RVE domain $\Omega$ is divided into two sub-domains $\Omega^{+}$and $\Omega^{-}$representing respectively the solid phase and the pore space, and $\Phi$ representing their interface. A negative value of the distance 

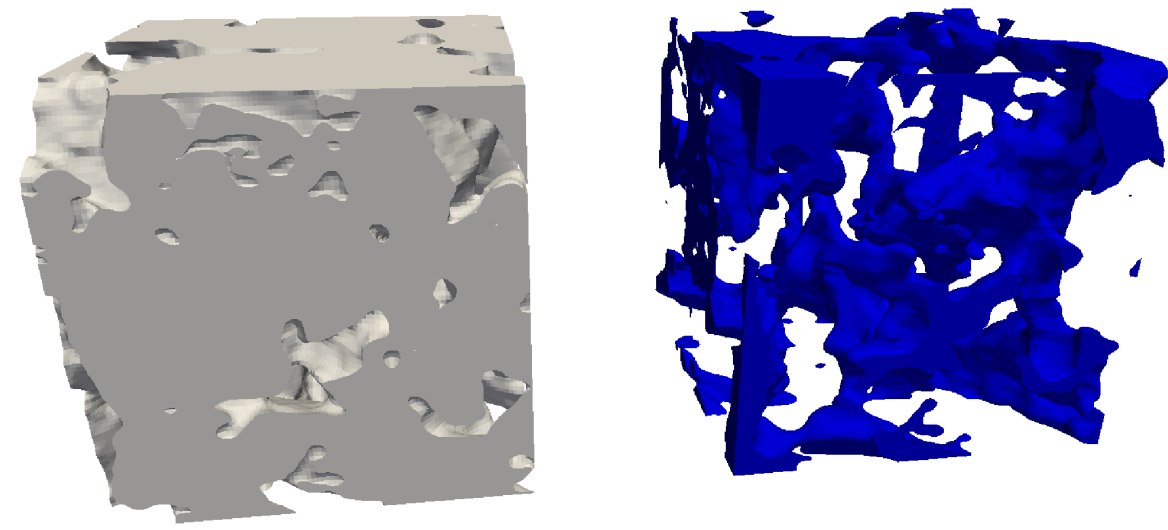

Fig. 4: Resulting segmentation of the solid part (left) and pore space (right) of the subset of sandstone micro-CT scan
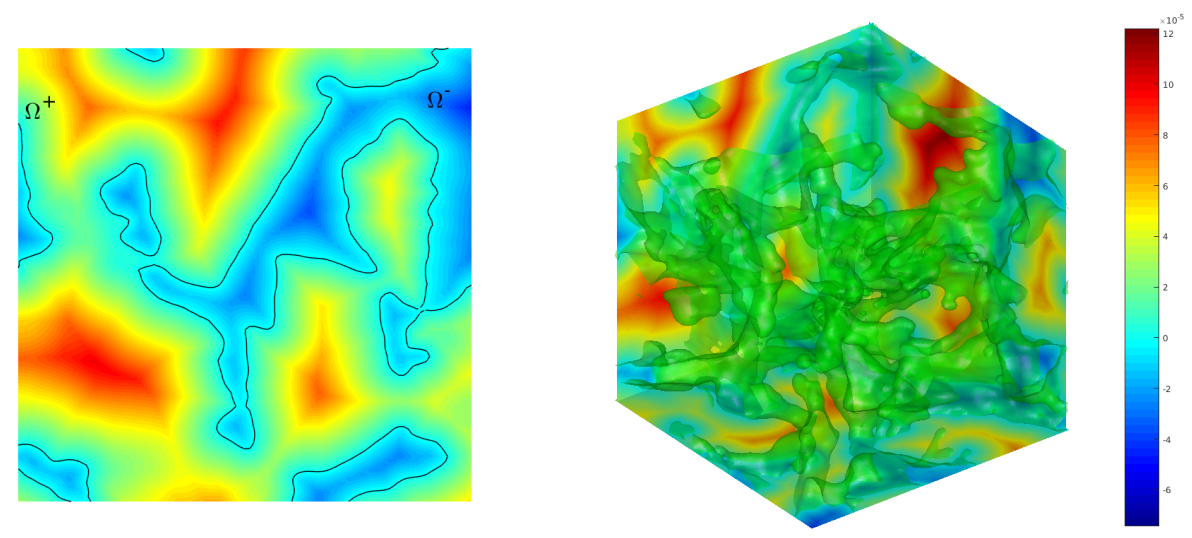

Fig. 5: Signed distance field of the sandstone sample where $\Omega^{+}$is the positive part corresponding to the solid part and $\Omega^{-}$the negative part corresponding to the porous space

function is attributed by convention to the domain $\Omega^{-}$as illustrated in Figure 5 . Practically, this distance function is evaluated on a discrete grid of points regularly placed on the spatial domain of interest of the scanned sample.

In order to maintain a sufficient accuracy without requiring excessive refinement of the background grid, the signed distance function is computed from an exact Euclidean signed distance field evaluation from discretized geometries [24] extracted with a Marching Cube process [34]. Other methods exist such as for instance the fast marching method [32] that features interesting execution times. However, it generally only yields an approximation of the signed distance, while the discretization processes require an accurate definition of the euclidean distance. Figure 5 right illustrates the signed distance field of the segmented micro-CT of the sandstone geometry obtained using an exact distance computation. This distance function will be used as a basis for the discretization procedures used in the next section. 


\section{Image-based modelling for heterogeneous geomaterials}

3.1 Conforming discretization of implicit geometries

The discretization approach explained here is based on the generation of conforming meshes for complex heterogeneous geometries, based on their implicit description by signed distance functions. The methodology was originally proposed by [21] for homogeneous structures and extended to heterogeneous structures by [22].

The conforming mesh generation strategy is subdivided into four main steps as follows :

1. Definition of a size function $h(\mathbf{x})$ based on specific geometrical characteristics

2. Generation of a nodes distribution based on an octree decomposition using this size function

3. Optimized surface meshing of the internal pore space surface and of the external structure boundaries (i.e. the external faces of the scan sample)

4. Optimized volume meshing based on a Constrained Delaunay Tetrahedralization (CDT) starting from the enclosed surface mesh generated during step 3.

\subsubsection{Size function definition}

The procedure starts by defining a size function $h(\mathbf{x})$, where $\mathbf{x}$ represents the spatial coordinates, that is used to control the elements sizes. Dealing with complex microstructures can quickly lead to a large number of degrees of freedom in the simulation process due to important mesh refinements. In fact, complex shapes of the microstructure motivate taking into account local features such as narrow gaps (nar), high curvatures (curv) or initial interface sizes (iis), thereby requiring a local increase of the nodes density. The size function is constructed based on the distance function to generate such non-uniform meshes refined only locally where it is necessary. The size function is thus the starting point of the optimization process and of the initial nodes generation. The minimum initial imposed size values $h_{0}$ depending on the three geometrical parameters iis, curv and iis are first computed as expressed in Equation (2). In the present context, a low sensitivity to curvature is used to avoid too large differences in the element sizes that can cause a conditioning problem of the stiffness matrix in FEM simulations.

$$
h_{0}(\mathbf{x})=\min \left(\left\{h_{i i s}(\mathbf{x}), h_{n a r}(\mathbf{x}), h_{c u r v}(\mathbf{x})\right\}\right)
$$

To avoid poor quality elements due to significant element size variations on small distances, a smooth evolution of the size function is enforced using a gradient limiting factor $g$ [35] expressed as

$$
\|\nabla h(\mathbf{x})\|=g
$$

Figure 6 illustrates the size function $h(\mathbf{x})$ of the segmented sandstone sample.

\subsubsection{Optimization by means of extended Persson-Strang truss analogy}

Dealing with complex geometries makes it difficult to reach an ideal mesh in which only quasi-regular tetrahedra are present. The objective of the optimization process is to minimize the magnitude of the difference between the lengths of the edges 


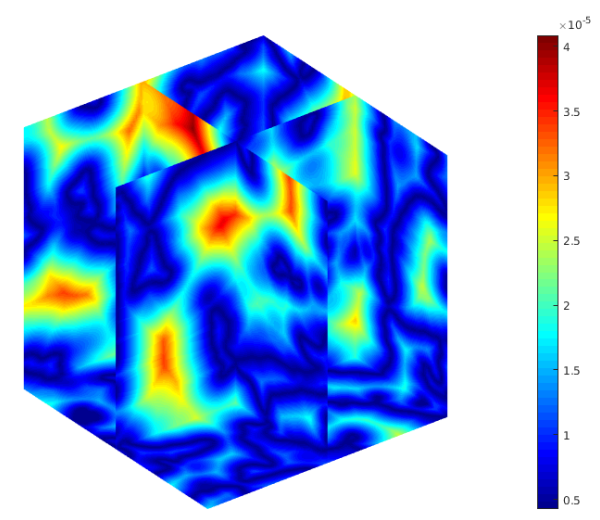

Fig. 6: 2D slices of size function $h(\mathbf{x})$ based on the segmented sandstone

in an element, and this over the entire mesh, in order to reach an optimal element quality for a given triangulation. This is achieved by finding an optimal configuration of the nodes positions for a given connectivity. The mesh quality optimization is based on an iterative technique that uses a simple mechanical analogy between the optimal mesh for a given connectivity and the equilibrium configuration of a 3D truss of elastic bars of stress-free lengths, or equivalently a structure made of springs [35]. In this analogy, the edges of tetrahedral elements and the mesh nodes correspond respectively to the bars and joints of a truss system. To this end, an attractive/repulsive force field $f\left(l, l_{0}\right)$ is defined on each bar, depending on its current length $l$ and its desired length $l_{0}$ set by the size function $h(\mathbf{x})$. This force field steers the nodes positions towards optimal elements shapes. By assuming an appropriate force-elongation relationship for the bars, the final nodes positions (x) for a set of fictitious forces $(\mathbf{F})$ can be found iteratively by solving for static equilibrium according to

$$
\mathbf{F}(\mathbf{x})=\sum_{i} \mathbf{F}_{i n t, i}(\mathbf{x})+\mathbf{F}_{\text {ext }, i}(\mathbf{x})=0
$$

where $\mathbf{F}_{\text {int }}$ and $\mathbf{F}_{\text {ext }}$ are respectively the internal forces present in the bars and the external forces coming from boundary constraints defined by fixing nodes on the boundary of the domain to be meshed (enclosing surface for a 3D mesh, enclosing contour for a surface mesh).

The fictitious force vector $\mathbf{F}(\mathbf{x})$ acting on each node depends on a changing topology of the truss system (i.e. if a new triangulation is produced during the node relocation process). This renders $\mathbf{F}(\mathbf{x})$ not continuous accross arbitrary $\mathbf{x}$ variations, thereby inducing difficulties to solve the system. When large node movements arise, such Delaunay retriangulations are indeed performed to improve the truss topology. The non-linear equations system can be simply resolved asymptotically by introducing an artificial time dependency (5) and using a forward first order Euler time integration scheme (6) starting from an initial Delaunay triangulation. Such a scheme is used until a stationary state is reached according to a pre-defined tolerance. The resulting fictitious mechanical equilibrium is achieved when the nodes movements tend to vanish. 
The following system of ordinary differential equations (ODEs) is considered (in non physical units), with the initial condition $\mathbf{x}(0)=\mathbf{x}_{0}$ being the initial node distribution :

$$
\begin{gathered}
\frac{d \mathbf{x}}{d t}=\mathbf{F}(\mathbf{x}), \quad t \geq 0 \\
\mathbf{x}\left(t_{n+1}\right)=\mathbf{x}\left(t_{n}\right)+\Delta t \mathbf{F}\left(\mathbf{x}\left(t_{n}\right)\right)
\end{gathered}
$$

Practically, the expression (7) is used for the internal repulsive/attractive forces field, allowing internal forces $\mathbf{F}_{\text {int }}(\mathbf{x})$ to reach the equilibrium state in a configuration matching the size function $h(\mathbf{x})$

$$
f\left(l, l_{0}\right)=k\left(l_{0}-l\right) \quad \text { where } k=\frac{l+l_{0}}{2 l_{0}} \approx 1
$$

To accelerate the convergence and get rid of slivers (zero volume elements) which may cause finite element computations to fail [36, 37, 35], an adapted ballvertex spring method [38, 39] is used during the optimization process by introducing additional (linear) springs on badly-shaped elements resisting the movements of a node of an element towards its opposite face in the element.

Each meshing step, namely the pore space surface meshing, the external structure boundary faces meshing and the 3D bulk meshing (of the solid phase and the pore space) implies an optimization process of the initial triangulation using this extended version of the principle of the described Persson-Strang truss analogy.

\subsubsection{Initial node distribution}

The mesh generation process starts with the initialization of an unconnected nodes distribution. A recursive refining process starting from the 8 corners of the structure is applied, in such a way that the local nodes density is related to the defined size function giving an octree decomposition [40,41, 42]. The advantage of such a method is to obtain a smoothly distributed initial nodes set controlled by $h(\mathbf{x})$, placing nodes close to their equilibrium position in the optimization process, and thereby reducing the number of retriangulations needed to get high quality FEM meshes.

\subsubsection{Surface Meshing}

The main difficulty with Delaunay-based mesh generators remains the enforcement of mesh conformity at material boundaries for heterogeneous materials, especially in 3D. In the present case this arises at the interface between the solid phase and the pore phase. Ensuring the conformity when nodes are present on both sides of the material boundaries before triangulation is a tricky task and is still a challenging problem both in theory and practice $[43,44]$. This is the reason that motivates the decomposition of the meshing process by meshing first the internal pore space surface and the external structure boundaries, in order to form the enclosed discretized surface that can be given as an input to $3 \mathrm{D}$ constrained Delaunay triangulation (CDT). As illustrated in Figure 7, a Marching Cube algorithm [34] is applied to extract the iso-surface corresponding to the internal 

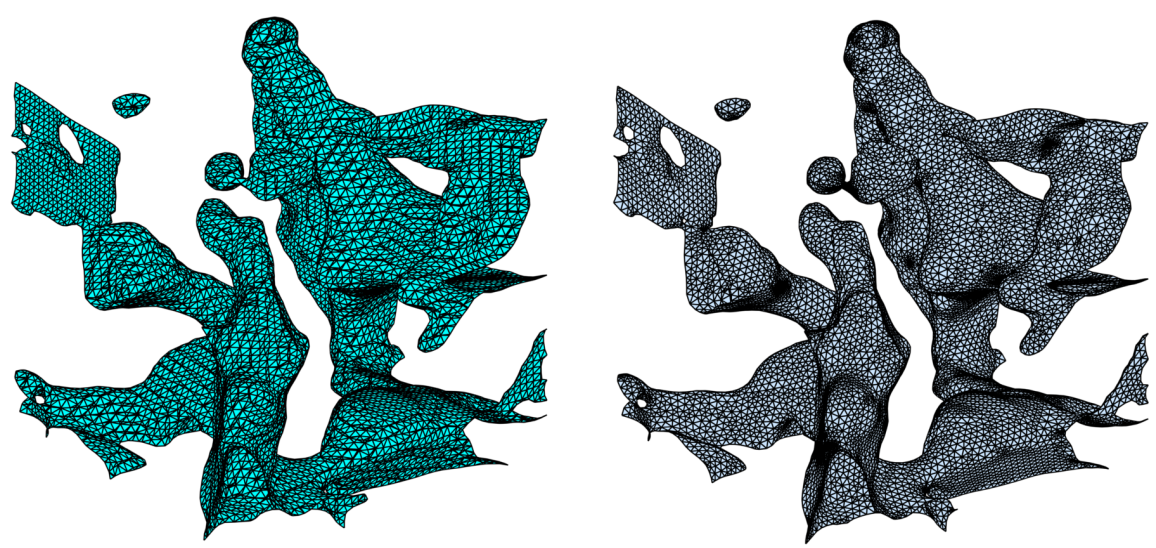

Fig. 7: Subset of the whole surface mesh - Left : Initial non optimized surface triangulation obtained by the Marching Cube algorithm [34], Right : Optimized mesh based on extended Persson-Strang truss analogy

pore space surface, giving an initial uniform ill-shaped surface triangulation (bad quality elements). This surface triangulation is then optimized by extended Persson truss analogy and refined locally according to the size function, generating a discretization appropriate for finite elements simulations.

Then, the 6 boundary faces of the sample microstructure are extracted, meshed and optimized with the extended Persson truss analogy, simplifying the problem by transforming the 3D boundary nodes on the six planes to deal with 2D problems. The external contours of material boundaries and the boundary nodes coming from the octree decomposition are extracted to mesh independently the 6 boundary faces as a 2D problem as illustrated in Figure 8.
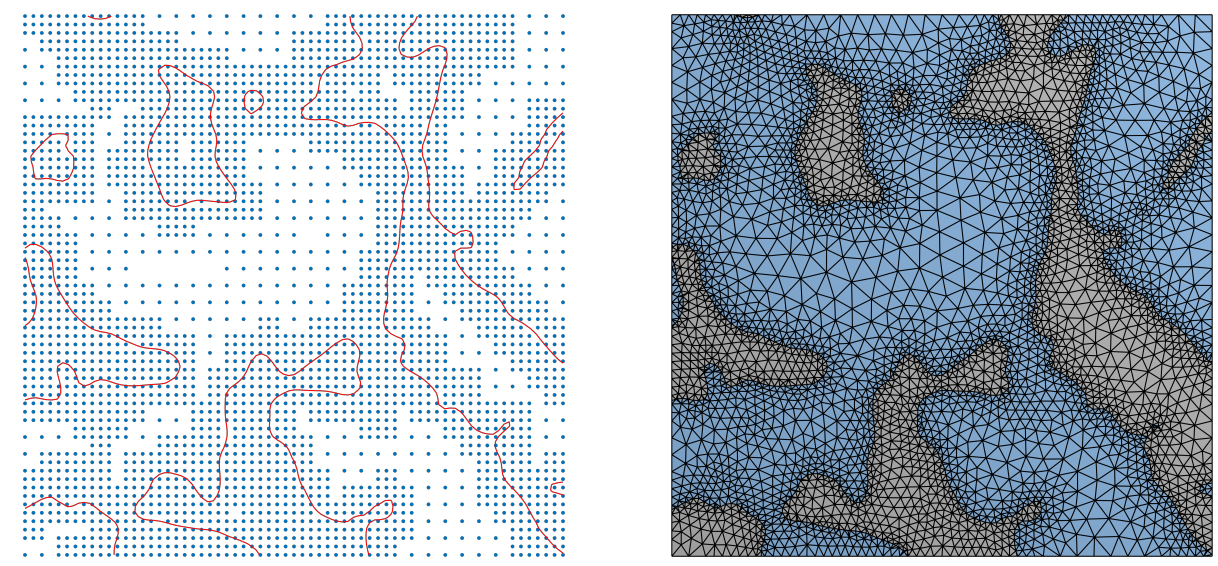

Fig. 8: Left : Extraction of external contours of sandstone sample and octree nodes belonging to the choosen face, Right : Optimized external structure boundary face mesh using extended Persson-Strang truss analogy 


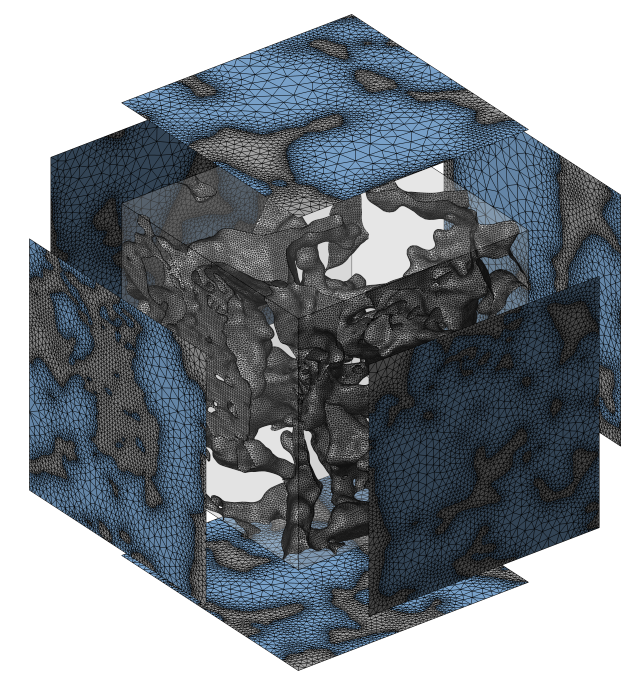

Fig. 9: Exploded view of merged internal material boundaries and external structure boundaries constituting the enclosed constrained facets taken as input in CDT

The process ends by merging the 6 optimized structure boundary faces meshes with the optimized internal pore space surface meshes; that will constitute the constrained facets providing a closed surface mesh to the CDT to ensure the mesh conformity as illustrated in Figure 9.

\subsubsection{Volume meshing}

The last step aims at generating the volume mesh for the solid phase volume and for the pore space volume, applying an efficient method that can generate boundary-conforming tetrahedral meshes such as CDT [45]. The constrained facets are given as input to the CDT module of the well-known and robust mesh generator TetGen [44], while the octree nodes distribution constitutes the background grid on which the CDT is applied. Finally, the extended Persson truss analogy process is applied to improve low quality elements appearing near the constrained facets due to the geometrical complexity and the imposed conformity. Figure 10 illustrates the optimized conforming mesh generated for the segmented sandstone sample.

\subsection{Embedded weak discontinuity models}

Regarding the problem of heterogeneous medium meshing, the most natural approach is to turn to conforming meshes as presented previously. Although being very efficient, the whole process may still suffer from poor quality elements for intricate geometrical configurations. More importantly, it may be difficult to consider evolving morphologies resulting of the development of cracks.

An alternative consists of building the approximation from non-adapted meshes. The main difference then lies in the fact that the nodes are positioned independently of the interfaces between the solid phase and the pore space. This avoids 

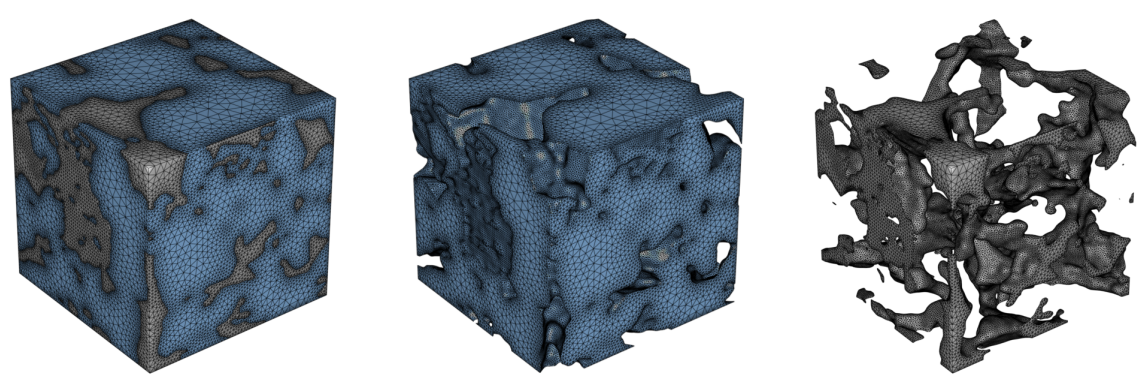

Fig. 10: Final optimized conform mesh of the RVE (\# Nodes=256 303/\# Elements=1 525 220) - Left : Global view of the optimized mesh for both phases (Solid phase/Pore space), Center : Solid phase meshed only, Right : Pore space meshed only

the need for relocating these nodes according to the heterogeneous geometry. This aspect is a major advantage towards some applications, such as probabilistic numerical studies with materials with a fine scale geometry that is not controlled. As a result of the use of a non conforming mesh, many elements - potentially all of them - are, for a given geometry, cut in two parts by a physical interface between the solid and the pore phase. Each of the two sub-domains of these elements defined by such an interface is included in a material phase, the properties of which are assumed to be known and different from the neighboring one. In order to allow these elements to represent this contrast of properties, it is necessary to enrich their kinematics by adding discontinuities in the interpolation of the strain field so-called "weak" discontinuities. Figure 11 shows a typical 4 noded element with such a discontinuity.

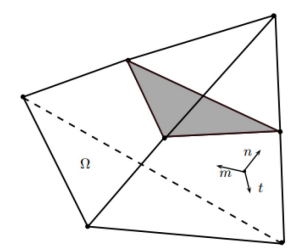

(a)

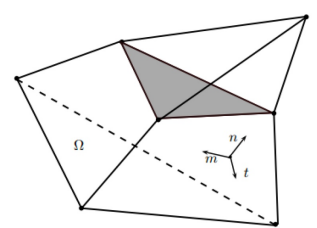

(b)

Fig. 11: Four noded element enriched by a strain discontinuity: left undeformed; right deformed.

Hence the kinematic enrichments constitute the keystone of this methodology. Originally developed to assess the problems posed by cracking in Finite Element models, such an enrichment is also advantageous in the context of the explicit consideration of heterogeneity. The methods of practical implementation of kinematic enhancements within a Finite Element model can be classified into two broad categories. Global approaches, on the one hand, are essentially based on the Partition of Unity. The enrichment then consists of the addition of interpolation functions 
and thus consists of adding global unknowns and global equations. The XFEM eXtended Finite Element Method [46] - belongs to this first category and has been used, for example, to describe three-dimensional arrangements of non-overlapping spheres [47]. An enrichment can be also be built, on the other hand, from a purely local point of view, leading to the family of methods called EFEM - Embedded Finite Element Method [48]. Also originally developed to tackle the problem of cracking - in particular the numerical localization induced by the use of softening laws - these approaches lead to an enrichment by means of local functions at the element level. Each of these added functions leads to an additional unknown and an additional scalar equation. The local character of those equations however makes it possible to condense them at the element level, thus preserving the size of the assembled discrete problem. Within this second family of methods of enrichment, the Method of Incompatible Modes is chosen here [49].

The theoretical framework of the Incompatible Modes Method is based on the three fields variational form from $\mathrm{Hu}$-Washizu [50]. In this form, the potential energy writes:

$$
\Pi_{H W}(u, \varepsilon, \sigma)=\int_{\Omega} \Psi(\varepsilon) \mathrm{d} \Omega-\int_{\Omega} \sigma \cdot\left(\varepsilon-\nabla^{s} u\right) \mathrm{d} \Omega-\int_{\partial \Omega_{v N}} u \cdot \bar{t} \mathrm{~d} S
$$

According to the basic principle of EFEM methods [51], the strain field $\varepsilon$ can be enriched according to the form $\varepsilon=\nabla^{s} u+\tilde{\varepsilon}$. This gives an expression depending on the displacement field $u$, the enrichment $\tilde{\varepsilon}$ and the stress field $\sigma$ :

$$
\Pi_{H W}(u, \tilde{\varepsilon}, \sigma)=\int_{\Omega} \Psi\left(\nabla^{s} u+\tilde{\varepsilon}\right) \mathrm{d} \Omega-\int_{\Omega} \sigma \cdot \tilde{\varepsilon} \mathrm{d} \Omega-\int_{\partial \Omega_{v N}} u \cdot \bar{t} \mathrm{~d} S
$$

The stationary character of $\Pi_{H W}$ leads to the three-field formulation of the enriched mechanical problem:

Knowing: $\quad \bar{t}: \partial \Omega_{v N} \rightarrow \mathbb{R}$

For all: $\quad u \in \mathbb{U}_{0}, \tilde{\varepsilon} \in L_{2}(\Omega)$ and $\sigma \in L_{2}(\Omega)$

Find: $\quad u \in \mathbb{U}, \quad \tilde{\varepsilon} \in L_{2}(\Omega)$ and $\sigma \in L_{2}(\Omega)$

Such that:

$$
\int_{\Omega} \nabla^{s} u \cdot \frac{\partial \Psi}{\partial \varepsilon} \mathrm{d} \Omega-\int_{\partial \Omega_{v N}} u \cdot \bar{t} \mathrm{~d} S+\int_{\Omega} \tilde{\varepsilon} \cdot\left(\frac{\partial \Psi}{\partial \varepsilon}-\sigma\right) \mathrm{d} \Omega-\int_{\Omega} \sigma \cdot \tilde{\varepsilon} \mathrm{d} \Omega=0
$$

A compact spatial discretization for each of these fields can be defined. The Incompatible Modes Method - from which its name derives - provides, for the enriched deformation field, a base of orthogonal functions in $L_{2}(\Omega)$ to the ones chosen for the interpolation of the stress field.

$$
\begin{aligned}
\nabla^{s} u^{h} & =B^{T} \cdot U \\
\tilde{\varepsilon}^{h} & =G^{T} \cdot v \\
\sigma^{h} & =P^{T} \cdot s
\end{aligned}
$$

This type of interpolation leads to two sets of - potentially nonlinear - equations to solve that can be grouped as a vector of residual equations $\mathbf{r}$. On the one hand, a set of global coupled equations $\mathbf{r}_{F E}$ is stemming from the FEM assembly operation; while on the other hand, a set of decoupled local equations $r_{e}$ associated with each 
of the new parameters of incompatible modes is introduced. These parameters are the additional unknowns associated with the enrichment of the kinematics.

$$
\mathbf{r}=\left[\begin{array}{c}
\mathbf{r}_{F E} \\
{\left[r_{e}\right]_{e}}
\end{array}\right]=\left[\begin{array}{c}
\int_{\Omega} B \cdot \frac{\partial \Psi}{\partial \varepsilon} \mathrm{d} \Omega-\int_{\partial \Omega_{v N}} N \cdot \bar{t} \mathrm{~d} S \\
{\left[\int_{\Omega} G^{e} \cdot \frac{\partial \Psi}{\partial \varepsilon^{e}} \mathrm{~d} \Omega\right]_{e}}
\end{array}\right]
$$

The key point of the Incompatible Modes Method lies in its local character, and thus in the $r_{e}$ decoupled equations. Even in a nonlinear case, their solving is very fast and the incompatible mode parameters can be easily condensed at the local level [52].

Using the Incompatible Modes Method, in order to construct the enrichments, the CT scan sample 3D image is projected on a tetrahedral unstructured mesh generated using the GMSH software [53]. This projection is a feature offered by the python library SPAM $[54,55,56,57]$. This allows identifying tetrahedra from each phase (solid phase and pore space) and the tetrahedra crossed by the interface that are enriched kinematically. For the considered scan, this results the model illustrated in Figure 12.

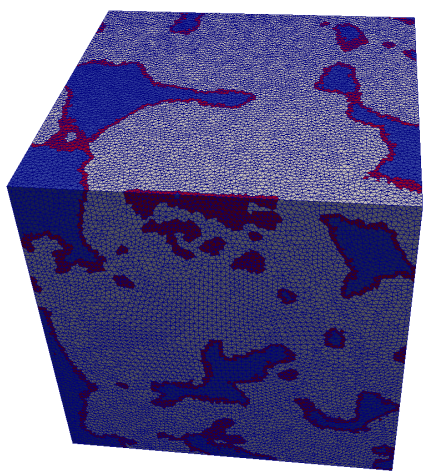

Fig. 12: Embedded weak discontinuity models (\# Nodes=234 996/\# Elements=1 329 159) obtained from the CT scan with solid elements (grey), pore space elements (blue), enriched elements (red)

\section{Comparison of FEM and EFEM methods based on a segmented sandstone sample}

To illustrate the ability of the models to conduct image-based finite element simulations, and to build the comparison between their results, the models derived from the CT scans are now subjected to two triaxial mechanical loading conditions as illustrated in Figure 13. First, a purely isotropic triaxial compression. The considered sample being isotropic (because of its limited size, unlike the overall material [23]), this matches an almost isotropic triaxial macroscopic strain state. 
Second, the sample is subjected to a deviatoric state to assess the magnitude of the local stress concentrations obtained in typical scenarii of triaxial testing.

Unlike in conforming finite element simulations, the Embedded Finite Element method requires considering a second phase in the pore space, described by solidlike constitutive laws. To comply with this requirement, the simulations use a second phase in the pore space with properties close to water (Poisson coefficient and the shear modulus tend towards 0.5 and 0 respectively). Preferring to avoid any consideration of fluid flow, the test is assumed undrained in the simulation.

The simulations are conducted with linear elastic materials. The model consists of a solid phase with properties $E=10 \mathrm{GPa}$ and $\nu=0.2$. The pore space is filled with a medium with properties $E=0.13 \mathrm{GPa}$ and $\nu=0.49$ to simulate the presence of an interstitial fluid (water). It is obvious that the values of the parameters used in simulations are somewhat arbitrary in the sense that they are values of macroscopic parameters used in a microscopic context. As a matter of facts, microscopic parameters in rock mechancis are difficult to identify and require advanced methods such as micro-indentation tests $[58,59]$ or micro-scratch tests [60] to determine them. Even with such tests, microstructural simulations are useful to derive values of actual model parameters and for interpretations, which motivates investigating the effect of their variation as performed in the sequel.

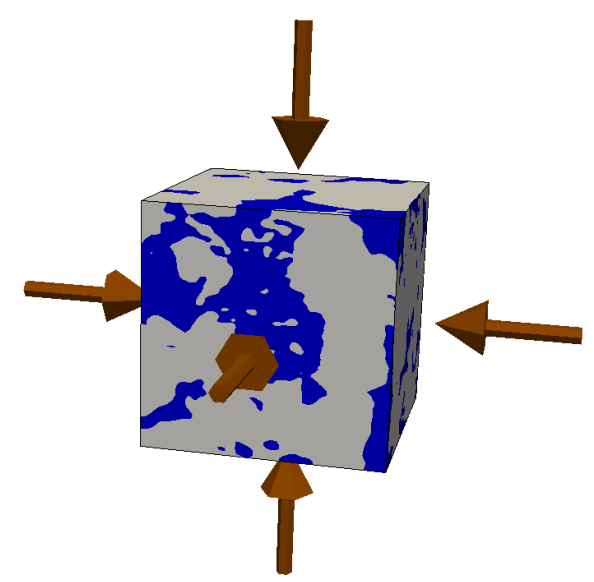

Fig. 13: Sandstone sample of size $[500 \mu m, 500 \mu m, 500 \mu m]$ subjected to a purely isotropic compression

For the interpretation of the numerical results, a classical Mohr-Coulomb failure criterion is considered, associated with a cut-off criterion for the tensile strength. In this composite criterion, the tensile cut-off aims at representing conditions giving rise to local cracking, while the Mohr-Coulomb part represents local conditions for the onset of local plasticity. It writes in $\sigma_{3}-\sigma_{1}$ plane:

$$
\left\{\begin{array}{l}
\text { compression } \sigma_{1}=K_{p} \sigma_{3}+\sigma_{c} \\
\text { tension } \sigma_{3}=-\sigma_{t}
\end{array}\right.
$$


in which $\sigma_{c}$ is the uniaxial compressive strength, $\sigma_{t}$ is the tensile strength and

$$
K_{p}=\frac{1+\sin \phi}{1-\sin \phi}
$$

where $\phi$ is the friction angle. With a Mohr-Coulomb failure criterion, the uniaxial compressive strength can be expressed in terms of the cohesion $c$ and friction angle as:

$$
\sigma_{c}=\frac{2 c \cos \phi}{1-\sin \phi}
$$

The macroscopic cohesion $c$ and friction angles $\phi$ of the Vosges sandstone are deduced from shear strength data obtained from triaxial tests and reported in [61]. They are equal to $10 \mathrm{MPa}$ and $37^{\circ}$ respectively. Those values are also consistent with the ones reported by $\mathrm{Hu}$ [23]. A uniaxial compressive strength $\sigma_{c}$ of $60 \mathrm{MPa}$ is therefore considered. No direct experimental data are available on the tensile strength of the Vosges sandstone. Recent efforts showed that the tensile strength values obtained by different techniques can differ significantly [62]. Practical estimates for the tensile strength of different sandstones were reported in [63], with a link to the uniaxial compressive strength given by

$$
\sigma_{t}=\frac{\sigma_{c}}{R}
$$

The value of the ratio $R$ was found to range from 8 to 26 (with exceptionally even larger values). As a result, there is an interest to consider different values for the post-processing of computational results in the sequel, and tensile strengths considered from 2 to $6 \mathrm{MPa}$ will be considered.

For the interpretation of the failure mechanisms, a plastic state parameter (PP) is computed locally based on the local stress state. It represents in a way the normalized distance to the nearest failure line of the composite criterion (MohrCoulom or cutoff). It writes:

$$
\mathrm{PP}= \begin{cases}\max (\underbrace{\left|\frac{\sigma_{1}}{K_{p} \sigma_{3}+\sigma_{c}}\right|}_{\mathrm{MC}} \underbrace{\frac{-\sigma_{3}}{\sigma_{t}}}_{\text {Tension }}) & \text { if } \sigma_{3}>-\sigma_{t} \\ \underbrace{\frac{-\sigma_{3}}{\sigma_{t}}}_{\text {Tension }} & \text { if } \sigma_{3} \leq-\sigma_{t}\end{cases}
$$

A local value above one of this ratio indicates that the onset of material nonlinearity is reached. A parametric variations of the plastic parameters $(\mathrm{PP})$ is equivalent to changing the properties of the material.

Only the solid phase of interest is reported on the figures of the next sections. For the embedded discontinuity model, the solid phase value of the enriched elements is plotted for the entire element for simplicity, hence the jagged aspect of the boundary.

Finally, the choice was made here to compare the methods based on models generating a comparable simulation cost. In other words, the assembly and system solving is made similar by using meshes containing comparable numbers of nodes (256300 and 235000 for FEM and EFEM respectively) and elements (1525000 and 1329000 for FEM and EFEM respectively). 


\subsection{Conforming model}

The application of a triaxial isotropic compression of $50 \mathrm{MPa}$ is first considered. This stress level matches the ones of classical tests [64]. This stress state is imposed by means of imposed deformations through applied kinematically uniform boundary conditions on the sample (uniform displacement of the model boundaries). The subset of the CT scan considered shows a isotropic behaviour, leading to a triaxial isotropic compression. Note that the base material is not fully isotropic [23], and the isotropy of the considered sample is caused by its limited size (lack of representativity).

A first analysis of the effect of heterogeneity induced by the presence of the pores can be performed by extracting the locations in the sample where tensile stresses are present (tensile stresses are considered here negative according to the usual convention in rock mechanics). Such stress states are represented in Figure 14. The left part of Figure 14 depicts the histogram of the most negative local principal stresses $\sigma_{3}$ in the mesh (lowest principal stress value when considering tensions as negative). The right part of Figure 14 represents the negative part of this histogram. It can be seen here that the presence of the pore space has a negligible effect on the presence of local tensions in the microstructure when considering a macroscopically isotropic stress state.
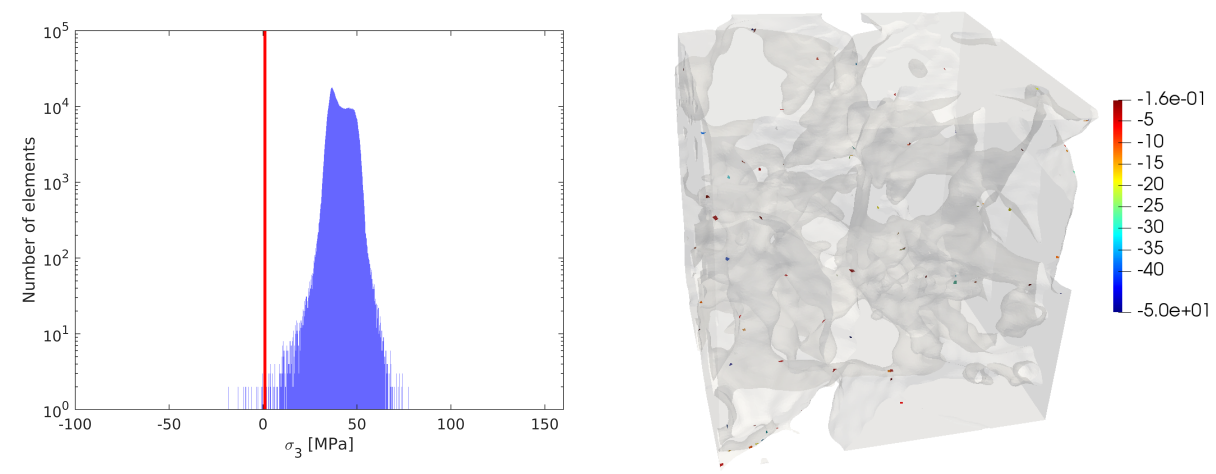

Fig. 14: Finite elements - Isotropic loading - Left : Histogram of $\sigma_{3}$ values obtained by FEM (red line isolates the tensile values), Right : Elements undergoing tension in the 3D volume predicted by FEM

The composite criterion (15) for the detection of the onset of cracking and plasticity can be further exploited to examine the local conditions for the presence of local material nonlinear response. Figure 15 left depicts the local stress states in the sample together with the assumed composite criterion for $R=15$ $\left(\sigma_{t}=4 \mathrm{MPa}\right)$. All points located outside the criterion are indicating that the cracking/plasticity onset is reached inside the microstructure. It can be seen that the macroscopic isotropic compression state (no macroscopic deviatoric stress) is translated into a set of stress states that significantly deviates from a isotropic stress state locally. For most of the points representing the stress states in this figure, the largest compressive principal stress exceeds the lowest compressive stress by a factor that ranges from 1 to 3 . Yet, the number of points exceeding the 
criterion remains rather limited. Most of the points located outside the criterion activate mainly the Mohr-Coulomb part of the criterion. Note that this assessment is purely based on the assumption that the fine scale material parameters values are comparable to the ones identified macroscopically. Figure 15 left also allows assessing to what extent a change of fine scale material strength parameters with respect to the macroscopic ones would affect the development of local plasticity. It can be seen from Figure 15 left that only a decrease of the angle of friction would significantly affect the number of stress state points exceeding the criterion. This is also reflected in Figure 15 right that represents the value of the local plastic state parameter (PP) according to the minor principal stress. The horizontal dashed line represents the threshold above which local failure/plasticity would be activated. From this figure, it can be seen that an important number of points are located above $\mathrm{PP}=0.5$.
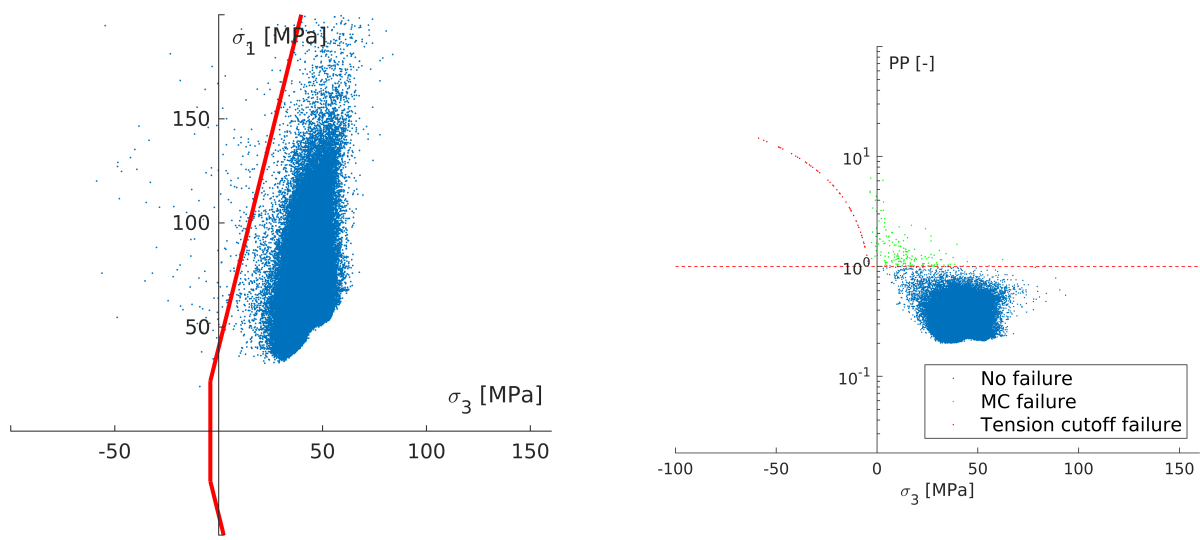

Fig. 15: Finite elements - Isotropic loading - Left: Stress state representative points in $\sigma_{3}-\sigma_{1}$ plane. In red, failure line expressed with a Mohr-Coulomb criterion with the parameters defined above $\left(c=10 \mathrm{MPa}, \phi=37^{\circ}\right)$ and with tensile cut-off at $\sigma_{t}=4 \mathrm{MPa}$, Right: Plasticity state parameter (PP) with points exceeding the tensile part of the criterion in green and the MohrCoulomb criterion in red
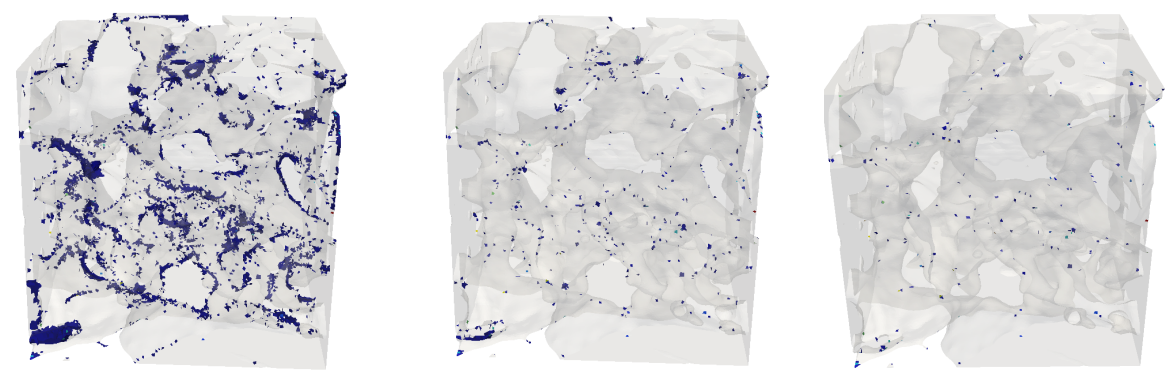

Fig. 16: Finite elements - Isotropic loading - Plasticity state parameter : PP $\geq 0.5$ (Left), $\mathrm{PP} \geq 0.7$ (Center), $\mathrm{PP} \geq 1$ (Right) 
Further illustrations of the results can be given in order to understand the link between the microstructural geometry and the presence of stress states for which the failure criterion is reached. To this end, the PP defined above is depicted on cuts in the sample in Figure 17, where a PP value of 1 is attributed to the points exceeding the composite failure criterion. Note that such higher values are also located in positions closer to the external faces of the sample as a result of the stiff boundary conditions applied.
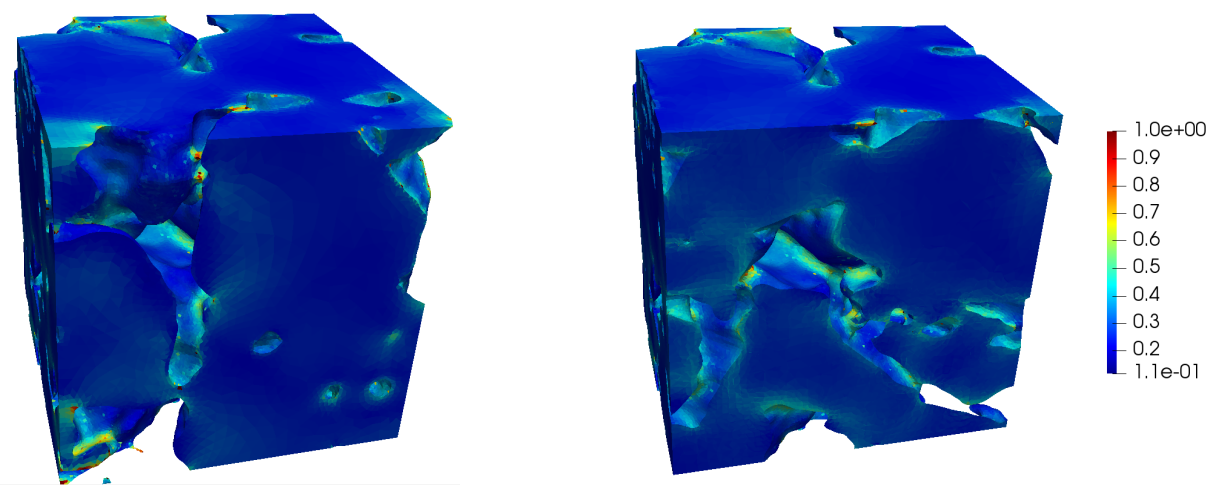

Fig. 17: Finite elements - Isotropic loading - Plasticity indicator PP in the 3D volume, for two cuts ( $x=0$ on the left and $x=82$ on the right) in the sample. Elements exceeding composite failure criterion match (in red) are characterised by a PP of 1.

As a second illustration, a triaxial loading with an important deviator is applied on the sample. Based on the data reported in [61], the loading considered is determined such that it matches a stress state at which the macroscopic failure is not yet reached, but for which an increase of the deviator would reach the macroscopic shear strength criterion in its linear part. This loading is taken as $\sigma_{1}=50 \mathrm{MPa}, \sigma_{2}=\sigma_{3}=20 \mathrm{MPa}$. To apply those boundary conditions, the principal (compressive) stress values are translated into principal (contraction) strain values, using the homogenized stiffness of the material. The homogenized stiffness of the material was obtained from simulations, and was assumed isotropic for the sample used here (homogenized values from computations showed a deviation of less than $2.3 \%$ with respect to isotropy for the stiffness components). This of course may have an impact on the isotropic or anisotropic nature of a real sandstone. But, as mentionned in Section 2, this subset was taken as such essentially for numerical considerations (i.e. computational cost), and not to assess the degree of isotropy of the material.

Figure 18 represents the local stress states in the sample for the above deviatoric macroscopic loading. Clearly, the deviatoric loading further expands the range of the local major principal stress. A significant number of representative points exceed the criterion, showing that plasticity and failure are locally extended within the sample. Note of course that the simulation remains elastic, and that stress redistributions have to be expected as from the first plastification. The corresponding spatial distribution of points having exceeded the criterion in the sample is given in Figure 19. It is clear from this figure that the local material nonlin- 


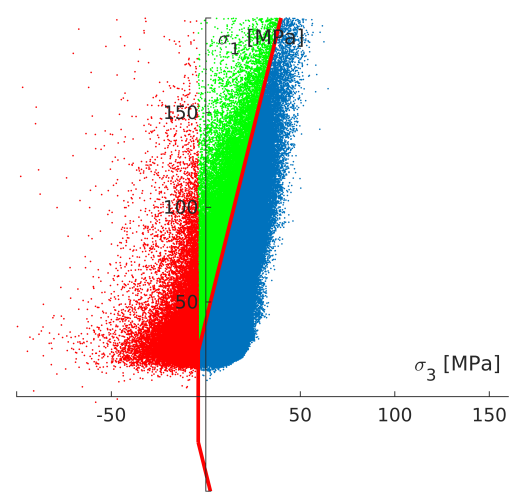

Fig. 18: Finite elements - Deviatoric loading - Stress state representative points in $\sigma_{3}-\sigma_{1}$ plane of the deviatoric case with elements activated by the tensile parts in red and MC parts in green. In red, failure line expressed with a Mohr-Coulomb criterion with the parameters defined above $\left(c=10 \mathrm{MPa}, \phi=37^{\circ}\right)$ and with tensile cut-off at $\sigma_{t}=4 \mathrm{MPa}$

earity is reached through both parts of the criterion. It also illustrates that the tension part of the criterion is increasingly reached for decreasing tensile strength as expected.
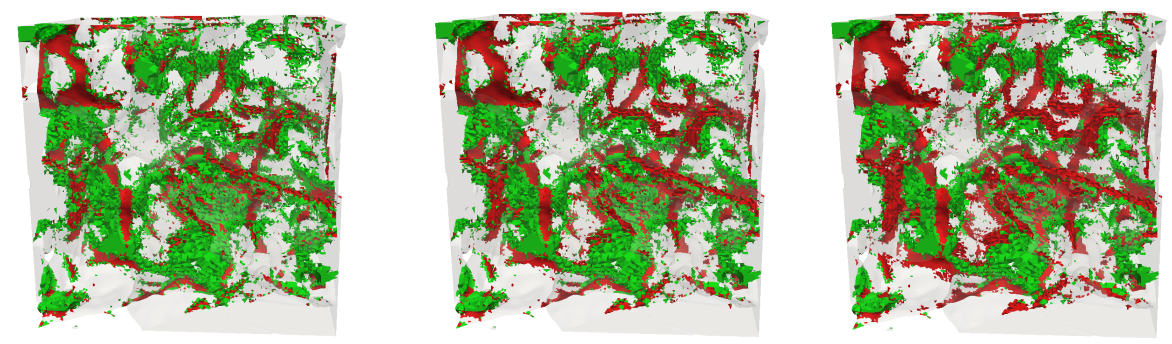

Fig. 19: Finite elements - Deviatoric loading - Elements where the PP is above 1 depending on whether the tensile (red) or MC parts (green) are activated for different value of $\sigma_{t}: 6 \mathrm{MPa}$ (Left), $4 \mathrm{MPa}$ (Center), $2 \mathrm{MPa}$ (Right)

\subsection{Embedded discontinuity model}

Like for the finite element model, the embedded discontinuity approach is first tested using the the triaxial compression case. This is achieved using the mesh presented in Figure 12.

For the isotropic compression, the histogram of the lowest principal stress values in the model is depicted in Figure 20. Unlike for the finite element case, no element in the mesh is found to be subjected to local tensile stresses. This difference may be related to the local level of discretization. The embedded discontinuity model makes use of a uniform element size, while the classical finite element model 
uses selective refinement/unrefinement depending on the local features of the geometry. In view of the limited amount of tensile stressed elements found based on the finite element model, this is however not a problematic difference.

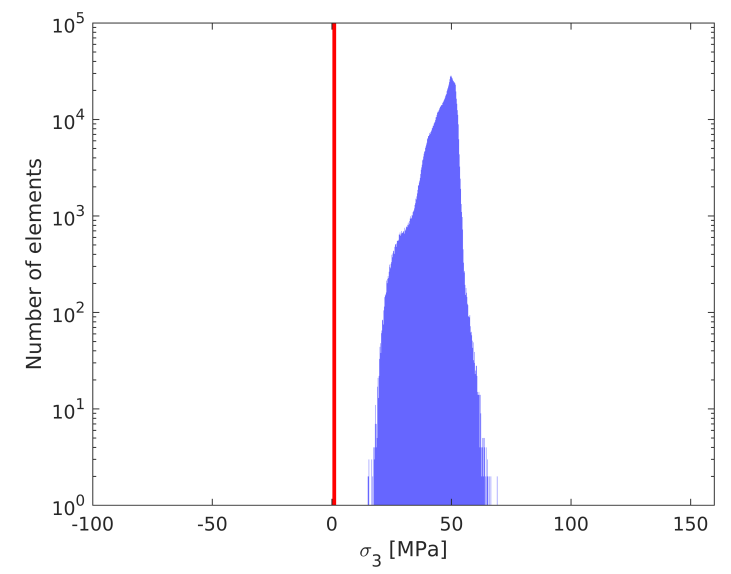

Fig. 20: Embedded discontinuity model - Isotropic loading - Histogram of $\sigma_{3}$ values obtained by the embedded discontinuity model

Points representing the stress states with respect to the assumed criterion are represented in Figure 21 left. This distribution of points in the $\sigma_{3}-\sigma_{1}$ space is quite different from the similar graph obtained by classical finite elements (Figure 15). With the embedded discontinuity model, the translation of the macroscopic isotropic compression state into local stress states exhibits a significantly lower deviation from a isotropic stress state. For most of the points representing the stress states in Figure 21, the largest compressive principal stress exceeds the lowest one by a factor that ranges from 1 to 2 (rather than 3 for the conforming finite element simulation). Like deduced from the finite element model, only a decrease of the angle of friction would significantly affect the number of stress state points exceeding the criterion, but in lower proportions than with the conforming finite element simulation. This is also reflected in Figure 21 right. The horizontal dashed line represents the threshold above which plasticity would be activated. From this figure, it can be seen that much lower values of PP should be considered to embed points representing stress states inside the sample. As a consequence, Figure 16 remains quite different from the equivalent figure for finite elements. Much less points are found in the range of $\mathrm{PP}$ above 0.7 , even though the figure corresponding to $\mathrm{PP}=0.5$ is rather similar to the classical finite element case.

These examples show that the model results differ due to their essential features. The classical finite element model is defined such that local geometrical features are captured by selective refinement. Small pores are strongly refined, while large solid components are discretized by coarser elements. Conversely, the embedded model has the objective to allow discretizing any microstructures (here any subset of a full CT scan) with the same uniform mesh. The small features are thus less well captured in the latter, while it shows a much improved flexibility 

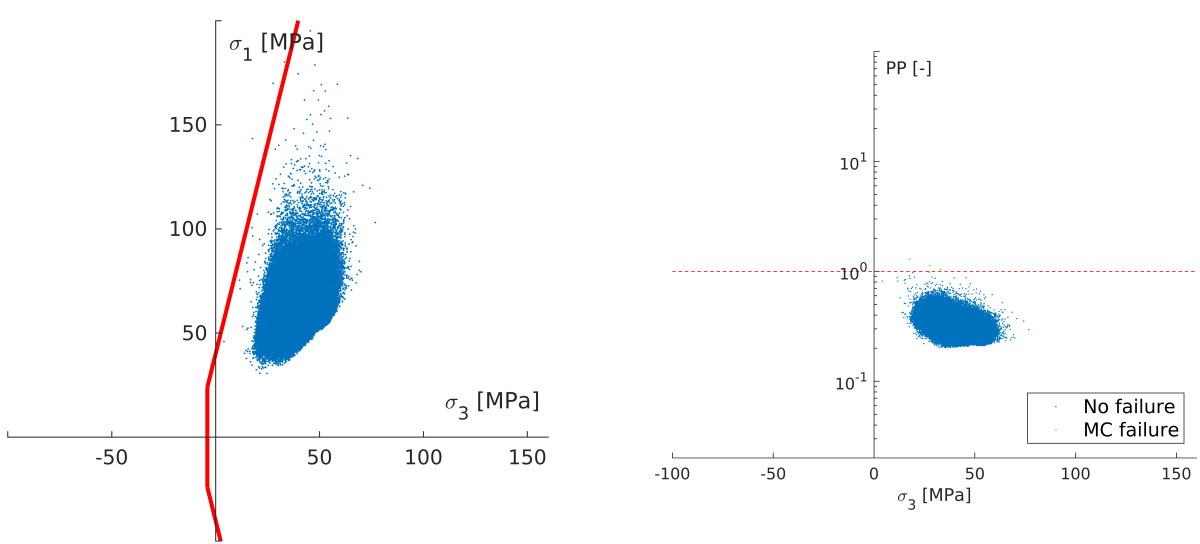

Fig. 21: Embedded discontinuity model - Isotropic loading - Left: Stress state representative points in $\sigma_{3}-\sigma_{1}$ plane. In red, failure line expressed with a Mohr-Coulomb criterion with cut-off in tension $\sigma_{t}=4 M P a$, Right: Plasticity state parameter (PP) with points exceeding the tensile part of the criterion in green and the Mohr-Coulomb criterion in red
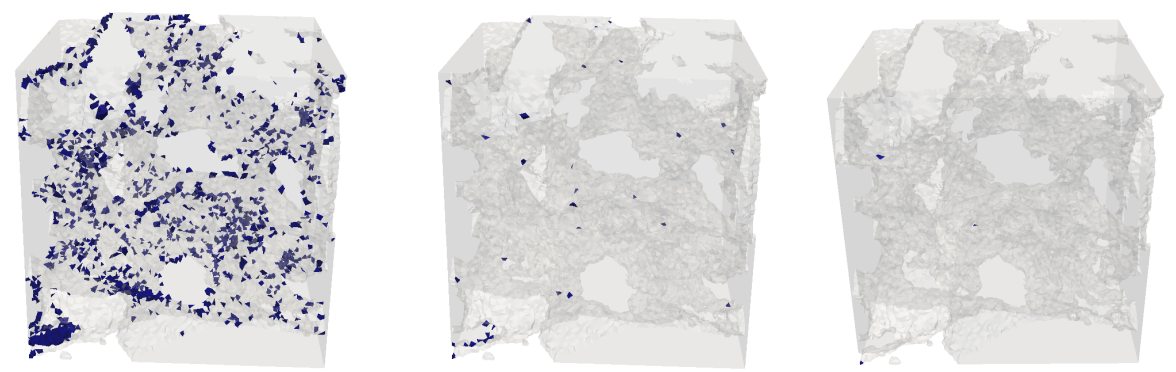

Fig. 22: Embedded discontinuity model - Isotropic loading - Plasticity state parameter : PP $>$ 0.5 (Left), $\mathrm{PP} \geq 0.7$ (Center), $\mathrm{PP} \geq 1$ (Right)

for studying many configurations with the same mesh. This is further illustrated when comparing the cuts given in Figure 23 with their conforming finite element counterpart in Figure 17.

When subjected to the same deviatoric loading as the classical finite element model, the identified trends are confirmed. Figure 24 represents the local stress states in the $\sigma_{1}-\sigma_{3}$ plane. Like for the isotropic compression case, the range of variation of the most compressive principal stress remains more restricted in the embedded discontinuity model than for classical finite elements, as can be appreciated by comparing Figure 24 with Figure 18. The number of points having exceeded the tensile part of the criterion is also much lower. This translates directly in the spatial distribution of points having exceeded the criterion represented in Figure 25. When comparing Figure 25 with Figure 19, it can be noticed that the tensile failure is much less detected in the embedded discontinuity model, irrespective of the level of tensile strength considered. Some detailed zones having reached the Mohr-Coulomb part of the criterion are also less detected (see for instance the top-left-back part of the sample in Figures 25 and 19). These latter 

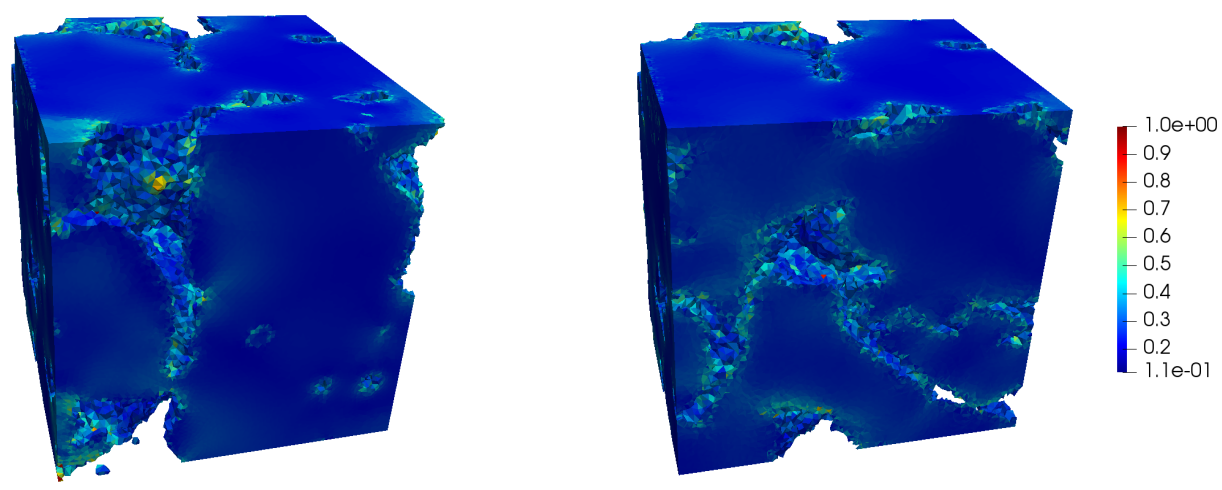

Fig. 23: Embedded discontinuity model - Isotropic loading - Plasticity indicator in the 3D volume, for two cuts ( $x=0$ on the left and $x=82$ on the right) in the sample. Points undergoing plasticity match (in red) are characterised by a PP of 1

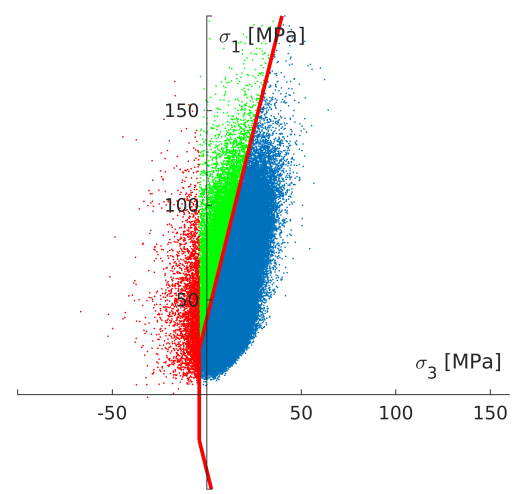

Fig. 24: Embedded discontinuity model - Deviatoric loading - Stress state representative points in $\sigma_{3}-\sigma_{1}$ plane of the deviatoric case with elements activated by the tensile parts in red and MC parts in green. In red, failure line expressed with a Mohr-Coulomb criterion with the parameters defined above $\left(c=10 \mathrm{MPa}, \phi=37^{\circ}\right)$ and with tensile cut-off at $\sigma_{t}=4 \mathrm{MPa}$

differences seem to be concentrated in the smallest pores, a fact that points again to the local refinement levels as explained above.

\subsection{Comparison of FEM and EFEM results}

To have a clearer comparison between the results of both models on detailed views, a cut of the $3 \mathrm{D}$ results is provided here for the lowest compressive stress and for the value of $\mathrm{PP}$.

For the isotropic loading case, Figure 26 compares the values of the lowest principal stress in a slice at $x=82$ for both methods, while Figure 27 compares the plasticity indicator PP. Some boundary effects appear at the surface of the solid phase in the embedded discontinuity model. They may be related to the use of strongly differing properties between the solid phase and the pore fluid-like phase. Yet, the stress distribution plot illustrates that the overall stress distribution 

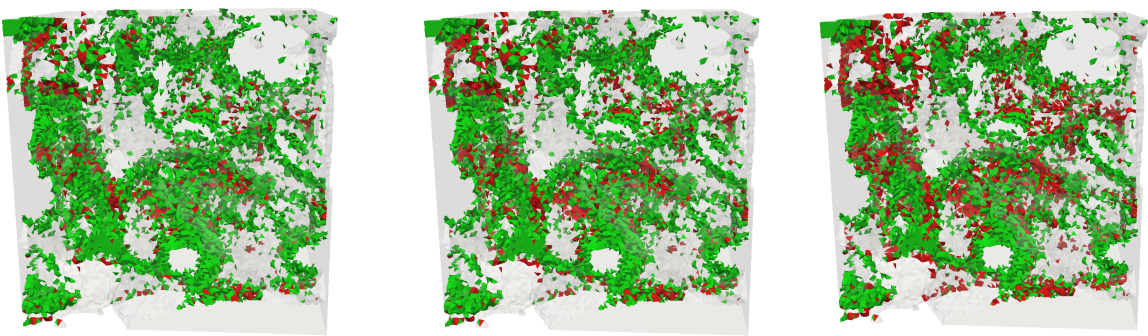

Fig. 25: Embedded discontinuity model - Deviatoric loading - Elements where the PP is above 1 depending on whether the tensile (red) or MC parts (green) are activated for different value of $\sigma_{t}: 6 \mathrm{MPa}$ (Left), $4 \mathrm{MPa}$ (Center), $2 \mathrm{MPa}$ (Right)
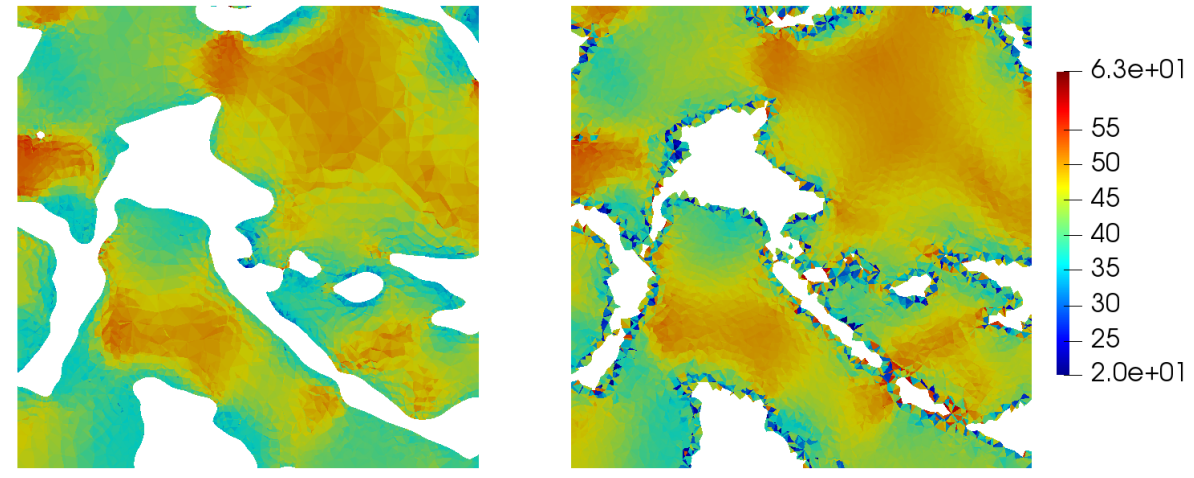

Fig. 26: FEM (left) and EFEM (right) results of $\sigma_{3}$ values on a slice in plane $x=82$ for the isotropic loading case. Only the solid phase is represented for the sake of clarity

is similar for both models, with only a more accurate estimation of the stress concentrations as a result of the selective mesh refinement in the classical finite element model. It can also be seen that the smallest pores (or the position at which the pore throats are smallest) are better reproduced by the classical finite elements for the same reason. An identical conclusion can be drawn from the map of the PP parameter. Note that as presented above, the levels of stress in this cut induced by the triaxial isotropic compression do not trigger plasticity/failure as indicated by the level of PP reached.

A similar set of figures are produced below for the deviatoric loading tested. For the isotropic loading case, Figure 28 compares the values of the most tensile principal stress in a slice at $x=82$ for both methods, while Figure 29 compares the plasticity indicator PP. Here also, the overall distributions of the stress field and the PP values are globally matching, with only deviations related to the selective refinement present in the finite element model, but not in the embedded discontinuity model. Note that the PP value color bar was capped to a unit value. Dark red values therefore include elements in which PP is larger than unity, and in which local failure/plasticity is indeed triggered. In these zones, the refined elements of the conforming finite element model have a clear impact on their extent with respect to the embedded discontinuity model. 

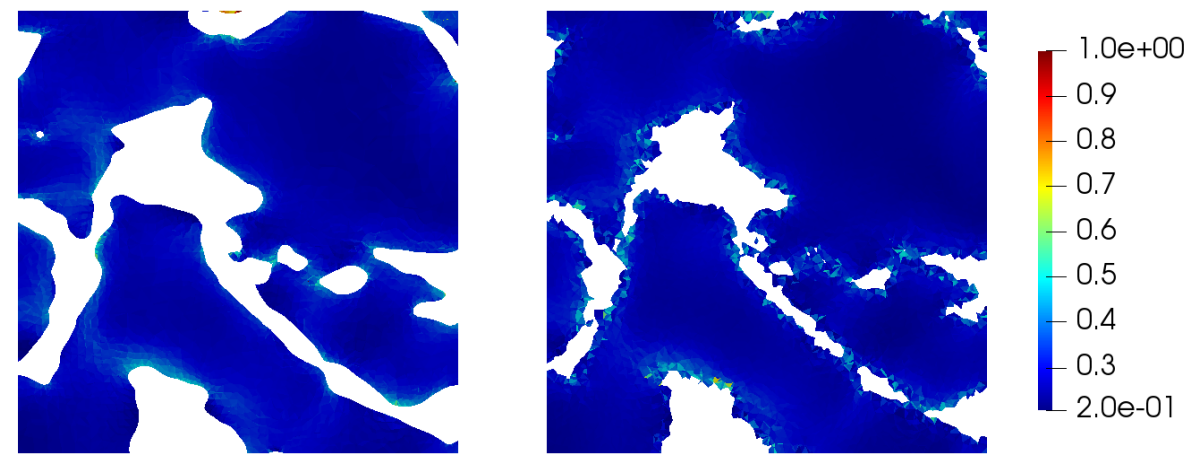

Fig. 27: FEM (left) and EFEM (right) results of PP values on a slice in plane $x=82$ for the isotropic loading case. Only the solid phase is represented for the sake of clarity
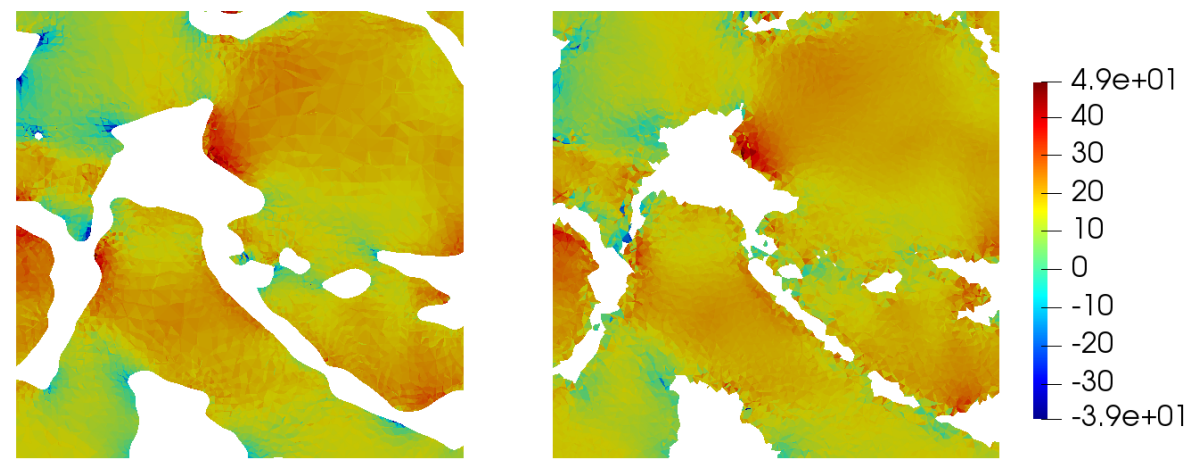

Fig. 28: FEM (left) and EFEM (right) results of $\sigma_{3}$ values on a slice in plane $x=82$ for the deviatoric loading case. Only the solid phase is represented for the sake of clarity
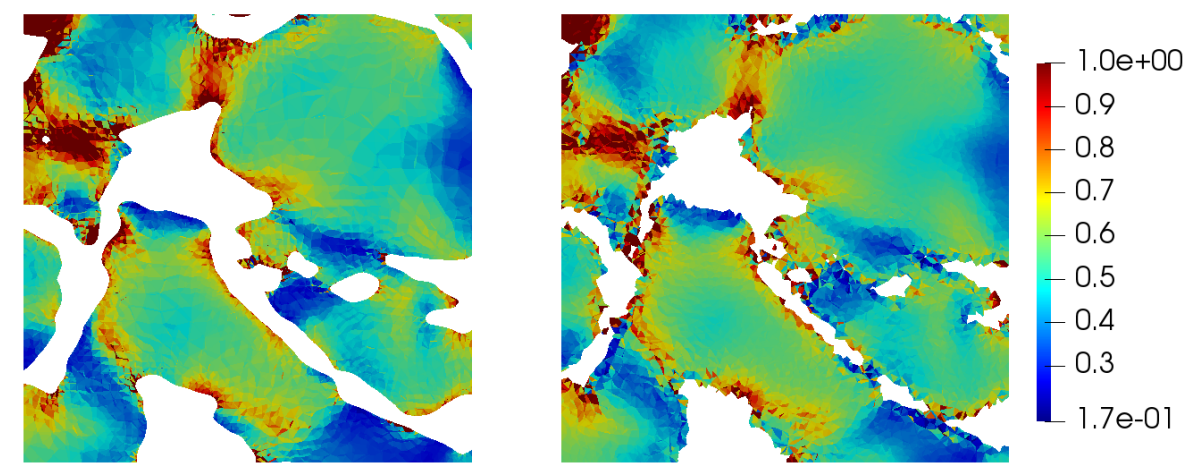

Fig. 29: FEM (left) and EFEM (right) results of PP values on a slice in plane $x=82$ for the deviatoric loading case. Only the solid phase is represented for the sake of clarity 


\section{Discussion}

The computations performed in this contribution based on a 3D image of a sandstone show that automated computational models can be set up to exploit raw CT scan data. The detailed comparison of two discretization schemes able to process such raw data showed that the overall behaviour can be captured by both approaches that deliver complementary features. The finite element mesh generation with selective refinement allows capturing detailed geometrical features of the microstructure. However, it requires a pre-processing stage to produce tetrahedral meshes with high quality elements. The embedded discontinuity-based models reproduce the overall mechanical effects. Being based on uniform size meshes, they are not designed to capture all the geometrical details, but present the advantage that their non conforming mesh can be projected on any microstructural geometry without change.

Next to these direct comparisons of results reached by both methods, some additional comments can be given, related to the selection of the methodology to use depending on the objectives targeted.

The first comment relates to the computational cost of the methods. The representation of the heterogeneous geometry by means of embedded weak discontinuities induces a marginal computational preprocessing cost that consists of the detection of elements cut by the boundary surface of the pore network. A simple unstructured mesh can be used, which allows efficient simulations pre-processing. The kinematic enrichment is computed at the element level, and can be condensed before assembly, which does not cause any additional cost in terms of the generated system of discretized equations. Post-processing would however require an additional implementation of tetrahedra cutting for vizualization purposes (not implemented here). With respect to these features, the generation of a conforming mesh incurs the pre-processing cost associated with the generation of the target size function, and with the mesh optimization step for surface meshes and 3D meshes. These operations can however be optimized to reach a situation in which the set-up of model could be reached in a few minutes. Secondly, the use of a conforming mesh with element sizes constrained by local features leads to rather fine discretizations with the associated assembly and solving cost. This should be balanced with respect to the intended accuracy, but more importantly requires a careful understanding of the effects of the size functions parameters (dependency of element sizes to curvatures, to narrowness of pore channels, etc.). Finally, postprocessing of the results is fully standard as a result of the use of regular finite element meshes. Existing standard or dedicated FE packages with existing dedicated constitutive laws and formulations can also be used.

Other features remain different between both methodologies as a result of the construction of the approximation. The embedded discontinuity methodology has the benefit of being extendable towards strong discontinuities for representing cracking. This is not the case for regular finite elements that require additional ingredients for the modelling of cracks (XFEM, embedded strong discontinuities, gradient regularization ...). Conversely, the consideration of an empty pore space (drained tests) can be more directly addressed by the conforming approach (no elements generated in the pore space) than by the embedded discontinuity approach that assumes the presence of a second phase in the definition of the discretization. A voided material therefore has to be approximated by a low stiffness material 
in the latter case, which may cause conditioning problems in the limit of a vanishing stiffness of the pore phase. Also, the extension to a quadratic interpolation of the displacement field is immediate for the conforming mesh generation (even though costly in terms of solving), while this is not straightforward in the case of embedded discontinuities.

From a physical viewpoint, the material properties of the solid phase were taken equivalent to the macroscopic material parameters as determined by [23] and by data from literature [61]. This choice can of course be debated, and as illustrated in Section 4, the resulting locations affected by tensile stresses can affect results. Any decrease of the tensile strength may have a considerable effect on the regions affected locally. The same reasoning holds to a lower extent for the Mohr-Coulomb part of the criterion. These facts bear significance for estimations of stress-induced permeability variations. As described in $[64,5]$, isotropic and deviatoric triaxial loading on rocks can lead to permanent alterations of the permeability, potentially as a result of local material nonlinearities induced by the heterogeneity associated with the pore space structure. The results illustrated here show that 3D imagebased models would allow investigating such potential microstructural effects.

\section{Conclusion}

This contribution presented the assessment of computational discretization strategies allowing to conduct automated simulations based on raw CT scan data for porous rocks with complex pore space morphologies. Both methods rely on pore and solid phase geometrical descriptions based on implicit functions and distance fields. Such a description can be obtained by level set-based segmentation techniques that were used here. Based on such implicitly described heterogeneous geometries, two discretization schemes were outlined. The first one makes use of the implicit description of the geometry to produce a conforming finite element discretization of the solid and pore phases. Based on the geometrical information, it allows generating selectively refined tetrahedral meshes to capture the complex geometry of the porous network and the corresponding solid boundaries. Complementarily, a second strategy based on a kinematic enrichment by incompatible modes is used to account for material boundaries based on a non-conforming mesh with uniform element sizes.

Mechanical simulations conducted on the CT scan pre-processed geometry show that the main feature of the behaviour can be captured by both methods. The conformal finite element procedure allows capturing the effect of more detailed geometrical features, while the incompatible mode-based framework is more flexible as it allows using the same (non conforming) mesh for potentially variable geometries. It is again emphasized that the simulations remain elastic, and that stress redistributions that have to be expected as from the first plastification cannot be captured by such simulations.

Future works are envisioned to exploit the benefit of both approaches. Some local geometrical information of the microstructure (translated as the target element size function defined in Section 3.1) could be used to produce tailored non conforming meshes with selective refinement by octree meshing at a marginal preprocessing cost. Finally all the tools that have been developed in this paper are adapted for simulations with a non linear mechanical behaviour of the solid phase 
(plasticity and tension cutoff), which allow considering the stress redistribution within the microstructure.

Acknowledgements The first author acknowledges the support of FRIA under Grant No. 29340757.

\section{References}

1. Shao J, Hoxha D, Bart M, Homand F, Duveau G, Souley M, Hoteit N (1999) Modelling of induced anisotropic damage in granites. International Journal of Rock Mechanics and Mining Sciences 36(8):1001 - 1012

2. Li J, Konietzky H, Frühwirt T (2017) Voronoi-based dem simulation approach for sandstone considering grain structure and pore size. Rock Mechanics and Rock Engineering 50(10):2749-2761

3. Li G, Liang ZZ, Tang CA (2015) Morphologic interpretation of rock failure mechanisms under uniaxial compression based on $3 \mathrm{~d}$ multiscale high-resolution numerical modeling. Rock Mechanics and Rock Engineering 48(6):2235-2262

4. Massart T, Selvadurai A (2012) Stress-induced permeability evolution in a quasi-brittle geomaterial. Journal of Geophysical Research 117:B07207

5. Massart T, Selvadurai A (2014) Computational modelling of crack-induced permeability evolution in granite with dilatant cracks. International Journal of Rock Mechanics and Mining Sciences 70:593 - 604

6. Hashemi MA, Khaddour G, François B, Massart TJ, Salager S (2014) A tomographic imagery segmentation methodology for three-phase geomaterials based on simultaneous region growing. Acta Geotechnica 9(5):831-846

7. Chen S, Yue ZQ, Tham LG (2006) Digital image based approach for threedimensional mechanical analysis of heterogeneous rocks. Rock Mechanics and Rock Engineering 40(2):145

8. Li CS, Zhang D, Du SS, Shi B (2016) Computed tomography based numerical simulation for triaxial test of soilrock mixture. Computers and Geotechnics $73: 179-188$

9. White JA, Borja RI, Fredrich JT (2006) Calculating the effective permeability of sandstone with multiscale lattice boltzmann/finite element simulations. Acta Geotechnica 1(4):195-209

10. Hashemi M, Massart T, Salager S, Herrier G, Franois B (2015) Pore scale characterization of lime-treated sandbentonite mixtures. Applied Clay Science 111:50 - 60

11. Yu Q, Yang S, Ranjith PG, Zhu W, Yang T (2016) Numerical modeling of jointed rock under compressive loading using x-ray computerized tomography. Rock Mechanics and Rock Engineering 49(3):877-891

12. Sufian A, Russell AR (2013) Microstructural pore changes and energy dissipation in gosford sandstone during pre-failure loading using x-ray ct. International Journal of Rock Mechanics and Mining Sciences 57:119 - 131

13. Raynaud S, Vasseur G, Soliva R (2012) In vivo ct x-ray observations of porosity evolution during triaxial deformation of a calcarenite. International Journal of Rock Mechanics and Mining Sciences 56:161 - 170 
14. Rabbani A, Jamshidi S (2014) Specific surface and porosity relationship for sandstones for prediction of permeability. International Journal of Rock Mechanics and Mining Sciences 71:25 - 32

15. Feng XT, Chen S, Zhou H (2004) Real-time computerized tomography (ct) experiments on sandstone damage evolution during triaxial compression with chemical corrosion. International Journal of Rock Mechanics and Mining Sciences 41(2):181 - 192

16. Kim KY, Zhuang L, Yang H, Kim H, Min KB (2016) Strength anisotropy of berea sandstone: Results of x-ray computed tomography, compression tests, and discrete modeling. Rock Mechanics and Rock Engineering 49(4):1201-1210

17. Sun W, fong Wong T (2018) Prediction of permeability and formation factor of sandstone with hybrid lattice boltzmann/finite element simulation on microtomographic images. International Journal of Rock Mechanics and Mining Sciences 106:269 - 277

18. Zhou XP, Xiao N (2017) A novel 3d geometrical reconstruction model for porous rocks. Engineering Geology 228:371 - 384

19. Zhou XP, Xiao N (2018) 3d numerical reconstruction of porous sandstone using improved simulated annealing algorithms. Rock Mechanics and Rock Engineering 51(7):2135-2151

20. Zhou XP, Xiao N (2018) A hierarchical-fractal approach for the rock reconstruction and numerical analysis. International Journal of Rock Mechanics and Mining Sciences 109:68 - 83

21. Persson PO, Strang G (2004) A simple mesh generator in matlab. SIAM Review 46:2004

22. Ehab Moustafa Kamel K, Sonon B, Massart TJ (2018) An integrated approach for the conformal discretization of complex inclusion-based microstructures. Computational Mechanics Submitted

23. Hu C, Agostini F, Skoczylas F, Jeannin L, Potier L (2018) Poromechanical properties of a sandstone under different stress states. Rock Mechanics and Rock Engineering

24. Sonon B, François B, Massart TJ (2012) A unified level set based methodology for fast generation of complex microstructural multi-phase rves. Computer Methods in Applied Mechanics and Engineering 223:103 - 122

25. Roubin E, Colliat JB, Benkemoun N (2015) Meso-scale modeling of concrete: A morphological description based on excursion sets of random fields. Computational Materials Science 102:183-195

26. Beucher S (2001) Geodesic reconstruction, saddle zones \& hierarchical segmentation. Image Analysis \& Stereology 20(3):137-141

27. Otsu N (1979) A threshold selection method from gray level histograms. IEEE Trans Systems, Man and Cybernetics 9:62-66

28. Caselles V, Kimmel R, Sapiro G (1997) Geodesic active contours. Int J Comput Vision 22(1):61-79

29. Chan TF, Vese LA (2001) Active contours without edges. Trans Img Proc 10(2):266-277

30. Shi Y, Karl WC (2008) A real-time algorithm for the approximation of levelset-based curve evolution. IEEE Transactions on Image Processing 17(5):645656

31. Bernard O, Friboulet D, Thevenaz P, Unser M (2009) Variational b-spline level-set: A linear filtering approach for fast deformable model evolution. IEEE 
Transactions on Image Processing 18(6):1179-1191

32. Osher S, Fedkiw R (2003) Signed Distance Functions, Springer New York, New York, NY, pp 17-22

33. Sethian J (1999) Level Set Methods and Fast Marching Methods: Evolving Interfaces in Computational Geometry, Fluid Mechanics, Computer Vision, and Materials Science. Cambridge Monographs on Applied and Computational Mathematics, Cambridge University Press

34. Lorensen WE, Cline HE (1987) Marching cubes: A high resolution 3d surface construction algorithm. SIGGRAPH Comput Graph 21(4):163-169

35. Persson PO (2005) Mesh generation for implicit geometries. PhD thesis, Massachusetts Institute of Technology, Cambridge, MA, USA, aAI0807802

36. Frey P, George P (2000) Mesh Generation: Application to Finite Elements. Hermes Science

37. Lo D (2015) Finite Element Mesh Generation. Taylor \& Francis

38. Bottasso CL, Detomi D, Serra R (2005) The ball-vertex method: a new simple spring analogy method for unstructured dynamic meshes. Computer Methods in Applied Mechanics and Engineering 194(39):4244 - 4264

39. Lin TJ, Guan ZQ, Chang JH, Lo SH (2014) Vertex-ball spring smoothing: An efficient method for unstructured dynamic hybrid meshes. Computers \& Structures 136:24 - 33

40. Legrain G, Cartraud P, Perreard I, Mos N (2011) An x-fem and level set computational approach for image-based modelling: Application to homogenization. International Journal for Numerical Methods in Engineering 86(7):915934

41. Macri M, De S (2008) An octree partition of unity method (octpum) with enrichments for multiscale modeling of heterogeneous media. Computers \& Structures 86(7):780 - 795

42. Paiva A, Lopes H, Lewiner T, Figueiredo LHD (2006) Robust adaptive meshes for implicit surfaces. In: 2006 19th Brazilian Symposium on Computer Graphics and Image Processing, pp 205-212

43. Si H (2010) Constrained delaunay tetrahedral mesh generation and refinement. Finite Elem Anal Des 46(1-2):33-46

44. Si H (2015) Tetgen, a delaunay-based quality tetrahedral mesh generator. ACM Trans Math Softw 41(2):11:1-11:36

45. Shewchuk JR (2002) Constrained delaunay tetrahedralizations and provably good boundary recovery. In: Proceedings of the 11th International Meshing Roundtable, IMR 2002, Ithaca, New York, USA, September 15-18, 2002, pp 193-204

46. Moes N, Dolbow J, Belytschko T (1999) A finite element method for crack growth without remeshing. International Journal for Numerical Methods in Engineering 46(1):131-150

47. Moes N, Cloirec M, Cartraud P, Remacle JF (2003) A computational approach to handle complex microstructure geometries. Computer Methods in Applied Mechanics and Engineering 192(28-30):3163-3177

48. Simo J, Oliver J, Armero F (1993) An analysis of strong discontinuities induced by strain-softening in rate-independent inelastic solids. Computational Mechanics 12(5):277-296

49. Ibrahimbegovic A, Wilson E (1991) Modified method of incompatible modes. Communications in Applied Numerical Methods 7(3):187-194 
50. K W (1982) Variational methods in elasticity and plasticity. Pergamon Press New York

51. Simo J, Rifai M (1990) A class of mixed assumed strain methods and the method of incompatible modes. International Journal for Numerical Methods in Engineering 29(8):1595-1638

52. Wilson E (1974) The static condensation algorithm. International Journal for Numerical Methods in Engineering 8(1):198-203

53. Geuzaine C, Remacle JF (2009) Gmsh: A 3-d finite element mesh generator with built-in pre- and post-processing facilities. International Journal for Numerical Methods in Engineering 79(11):1309-1331

54. E Andó, Cailletaud R, Roubin E, Stamati O, Wiebicke M, Couture CB, Matsushima T, Okubadejo O, Bertoni F, Sun Y, Colliat J (2019) Spam: Software for Practical Analysis of Materials A Python toolkit built on Numpy and Scipy. In preparation

55. Reinhard Furrer, Sain SR (2010) Spam: A sparse matrix R package with emphasis on MCMC methods for Gaussian Markov random fields. Journal of Statistical Software 36(10):1-25

56. Florian Gerber, Furrer R (2015) Pitfalls in the implementation of Bayesian hierarchical modeling of areal count data: An illustration using BYM and Leroux models. Journal of Statistical Software, Code Snippets 63(1):1-32

57. Florian Gerber, Moesinger K, Furrer R (2017) Extending R packages to support 64-bit compiled code: An illustration with spam64 and GIMMS NDVI3g data. Computer \& Geoscience 104:109-119, DOI 10.1016/j.cageo.2016.11.015

58. Randall NX, Vandamme M, Ulm FJ (2009) Nanoindentation analysis as a twodimensional tool for mapping the mechanical properties of complex surfaces. Journal of Materials Research 24(3):679690, DOI 10.1557/jmr.2009.0149

59. Mahabadi O, Randall N, Zong Z, Grasselli G (2012) A novel approach for micro-scale characterization and modeling of geomaterials incorporating actual material heterogeneity. Geophysical Research Letters 39:1303-, DOI 10.1029/2011GL050411

60. Akono AT, Miguel Reis P, Ulm FJ (2011) Scratching as a fracture process: From butter to steel. Physical review letters 106:204302, DOI 10.1103/PhysRevLett.106.204302

61. Besuelle P, Desrues J, Raynaud S (2000) Experimental characterisation of the localisation phenomenon inside a vosges sandstone in a triaxial cell. International Journal of Rock Mechanics and Mining Sciences 37(8):1223 - 1237

62. Perras MA, Diederichs MS (2014) A review of the tensile strength of rock: Concepts and testing. Geotechnical and Geological Engineering 32(2):525-546

63. Cai M (2010) Practical estimates of tensile strength and hoek-brown strength parameter mi of brittle rocks. Rock Mechanics and Rock Engineering 43(2):167-184

64. Selvadurai A, Glowacki A (2007) Permeability hysterisis of limestone during isotropic compression. Groundwater 46(1):113-119 